\title{
Higher-Order Free Logic and the Prior-Kaplan Paradox
}

\author{
Andrew Bacon, John Hawthorne and Gabriel Uzquiano
}

April 11, 2016

\section{Introduction}

A central theme from Modal Logic as Metaphysics is the idea that higher-order logic is a fruitful framework for formulating and assessing some of the somewhat elusive debates in the metaphysics of properties and modality. It is clear from reading the book that the choice to theorise in higher-order logic is not incidental to Williamson's project, but rather a substantial theoretical commitment. Within this framework certain compelling principles can be formulated, which, if taken to be about first order entities, would straightforwardly collapse into paradox; this is true, for example, of the comprehension principle that plays a central role in motivating higher-order necessitism.

However, Williamson's version of higher-order logic is not obviously a safe haven when it comes to the paradoxes. Indeed, this was something early proponents of higher-order logic were well aware of, and this was a central theme in the philosophy of higher-order logic in the early twentieth century. There were roughly two main schools of thought concerning the proper way to regiment a higher-order language. On a broadly Fregean picture, there's a tight connection between grammatical categories and the type theoretic hierarchy. There is a type $e$ to which individual constants and variables belong, and whenever $\tau_{1}, \ldots, \tau_{n}$ are types, there is a type of $n$-ary relations, $\left(\tau_{1}, \ldots, \tau_{n}\right)$, whose arguments are of type $\tau_{1}, \ldots, \tau_{n}$ respectively. Note that propositions can be thought of as 0-ary relations, which in this notation would be written (). Thus, for example, monadic predicates like 'is happy' have type $(e)$, unary connectives like 'it's not the case that' have type $(())$, binary connectives like 'and' have type $((),())$, first-order quantifiers like 'most people' have type $((e))$, and quantifiers into sentence position have type $((()))$.

As the last two examples suggest, each logical type in this sense can have its own kind of universal quantifier, which binds variables of that type. Whereas first-order quantifiers simply range over eligible referents for singular terms in the intended interpretation, it's important not to think of higher-order quantifiers as ranging over another domain of entities. For example, it wouldn't do to think of quantification into predicate position as quantifying over eligible 'referents' for predicates. Predicates do not refer, they predicate, and they cannot occupy the position of a singular term. It would be as much of a mistake to put a second-order quantifier in the place of a singular term, as to put a predicate in the place of a singular term. Broadly speaking this is the framework that Williamson is employing, in line with a venerable tradition tracing back to the likes of Frege, Church, Montague and Prior. 
By contrast, there's another school of thought, often attributed to Russell, in which each relational type is further stratified into levels indexed by the natural numbers. ${ }^{1}$ That is, instead of a single type of relations $\left(\tau_{1}, \ldots, \tau_{n}\right)$, for any ramified types, $\tau_{1}, \ldots, \tau_{n}$, there is a whole hierarchy of relations of different levels, written $\left(\tau_{1}, \ldots, \tau_{n}\right) / k$ for $k \in \mathbb{N}^{2}{ }^{2}$ So instead of a single type of proposition, we have infinitely many ramified propositional types, ()$/ 0,() / 1,() / 2$ and so on. One way to think of these types, borrowing a metaphor from Kaplan [11], is to think of the zero level propositional type as containing only propositions concerning the distribution of earth, wind, fire and water. The first level propositional type then concern matters of earth, air, fire and water, and matters concerning propositions about earth, air, fire and water (i.e., matters concerning zero level propositions). This generalizes in the obvious way: at any stage, a new level can be generated in an analogous way. For each of these ramified propositional types there is a universal quantifier. Note, however, that unlike the Fregean, the Russellian typically treats certain parts of the logical vocabulary as syncategorematic; negation, conjunction and even the quantifiers are not assigned a ramified type, but $\forall v \phi, \neg \phi, \phi \vee \psi$ are all well formed formulas provided $\phi$ and $\psi$ are of some propositional type and $v$ is a variable of any type. ${ }^{3}$ When we combine the universal quantifier with a propositional variable of ramified type ()$/ 0$ can achieve the effect of quantifying over 0 level propositions: in general the expression $\forall p_{n}$, where $p_{n}$ is of ramified type ()$/ n$, quantifies over propositions of level $n$.

This picture does not sit particularly well with the principle of universal instantiation applied at any level (hence the restriction in Church [4] p. 751). For example, at sentence level, this principle allows us to move from a universal generalization like $\forall p A$ to any instance $A(B / p)$, where $B$ could be any sentence:

$$
\forall p A \rightarrow A(B / p)^{4}
$$

Notice that for the stratifier this principle is doubly schematic in that $\forall p$ is a place holder for a quantifier of a particular level as well as having $A$ and $B$ as a place holders for any formula. For example, it could be that every 0 -level proposition satisfies $A$ (maybe $A$ is $\exists_{0} q p=q$, namely, being a 0-level proposition) but not every 1-level proposition does. So, if $B$ expresses a 1-level proposition, then it will not satisfy $A$. Logics that relax the principle of universal instantiation fall under the umbrella term 'free logic'. Some versions of free logic accept restricted instances of universal instantiation - for example, negative free logics characteristically claim that universal generalisations of atomic formulas imply their instances - but all relinquish unqualified UI.

There is in fact a more principled reason why the Russellian cannot keep the principle of universal instantiation: given any two quantifiers into sentence position satisfying the principle of universal instantiation, and some fairly uncontroversial background logic, one can prove that those quantifiers are logically equivalent. If $\forall$ and $\Pi$ are two quantifiers taking sentence position satisfying UI, then one can prove $\forall p A \leftrightarrow \Pi p A .{ }^{5}$ This argument is a generalisation of a theorem

\footnotetext{
${ }^{1}$ See Russell [22], Ramsey [21], Kripke [12], and Kaplan [11], Tucker and Thomason [26].

${ }^{2}$ Note that there are many different ways of implementing the ramificationist approach (it's very natural to only allow types $\left(\tau_{1}, \ldots, \tau_{n}\right) / k$ where $k$ is greater than the levels of $\left.\tau_{1}, \ldots, \tau_{n}\right)$. In what follows we shall be following Church [4] in considering only cumulative ramified type theories: informally a relation that accepts arguments of a given level, accepts arguments of any lower level. For the connection between this and the various versions of Russell's original theory see footnote 2 of [4].

${ }^{3}$ We assume that applying the logical connectives does not take us to a higher level.

${ }^{4}$ Provided $B$ contains no free occurrences of $p$, and no free variables that would become bound if substituted for $p$ in $A$; in what follows we will take this condition to be tacitly assumed.

${ }^{5}$ Without loss of generality we give the proof of one direction (up to a change of variables):

1. $(\forall p A \rightarrow A(q / p))$ (instance of UI for $\forall$.)

2. $\Pi q(\forall p A \rightarrow A(q / p))$ (by universal generalisation for П.)

3. $\forall p A \rightarrow \Pi q A(q / p)$ (by the principle $\Pi p(A \rightarrow B) \rightarrow(A \rightarrow \Pi p B)$ when $p$ is not free in $A$.)
} 
due to Harris [9]. While Harris was primarily concerned with first order quantifiers, his result is general and applies to quantifiers into other grammatical categories, assuming analogous principles of UI. ${ }^{6}$ Given UI, any ramification in any grammatical category would be pointless as all the different quantifiers in that category would collapse into one.

It's worth noting that if universal instantiation is relinquished then the following principle of existential generalisation will have to be given up as well:

$$
A(B / p) \rightarrow \exists p A
$$

This principle is equivalent to UI - this fact can be easily be seen by taking the contrapositive of the $\neg A$ instance of UI, $\neg \neg A(B / p) \rightarrow \neg \forall p \neg A$, eliminating double negations and applying quantifier duality. Without this rule one cannot infer from the fact that I'm thinking that snow is white that there is something I'm thinking.

The Russellian picture is supposed to help with a family of paradoxes, though we will in this paper be focusing on a particular species of paradox. As we will become clear, the rejection of UI is the key moving part to the Russellian style response to at least that species of paradox. Indeed, the principal virtues of this response can be articulated within a theory containing only one quantifier abiding by a restricted version of UI. ${ }^{7}$

Williamson, on the other hand, is primarily concerned not with the intensional paradoxes but rather with the metaphysics of modality as revealed by the interaction of various higher-order quantifiers with the modal operators within a broadly Fregean framework. While Russell-style theorists are forced to abandon UI, the Fregean is free to adopt or reject it. One central theme in Williamson's book is to connect this choice point with the higher-order necessitistcontingentist dispute. Let us focus on the principle of universal instantiation for quantification into sentence position. From this principle one can prove a necessitist principle for propositional quantification, which we can formalize as $\square \forall p \square \exists q p \equiv q$. Here $\equiv$ could stand for propositional identity, or simply necessary equivalence: however $\equiv$ is interpreted, all one needs to prove this result is UI, universal generalisation, the principle of necessitation plus the logical truth of the principle $p \equiv p .^{8}$

There hasn't been much work done to connect the debates about contingentism and necessitism with the literature on the intensional paradoxes. However, the principle of universal instantiation plays a pivotal role in both disputes, giving us reason to think that there are interesting connections to be drawn. It bears emphasis that both the Russellian stratifier and the contingentist reject UI, and ipso facto, go in for some kind of free logic. In this paper we're going to explore whether a free logic motivated by certain intensional paradoxes can undermine the case for necessitism. While necessitism can be formally reconciled with a free logic of this sort, many of Williamson's key abductive arguments for necessitism will not survive in this setting. This is because those arguments rely on logical explanations of certain data which depend on UI and Williamson's favoured comprehension principles, which aren't available within such a free logic.

Section 2 introduces our focal kind of paradox and explains why a free logic can offer a distinctive kind of solution. Section 3 makes vivid the relevance of universal instantiation to the higher-order necessitist/contingentist debate. Section 4 offers a number of free logical models

\footnotetext{
${ }^{6}$ See also McGee [27] for a discussion of the second-order variant.

${ }^{7}$ It's arguable that the rejection of UI is also the key part to a Russell-style treatment of many other paradoxes, but we shall not defend the broader claim here.

${ }^{8}$ Suppose that $p \equiv p$. Then by EG, we can infer $\exists q p \equiv q$. We then have $\square \exists q p \equiv q$ by necessitation, $\forall p \square \exists q p \equiv q$ by universal generalisation, and finally $\square \forall p \square \exists q p \equiv q$ by necessitation again.
} 
for a family of claims that threaten paradox within the classical setting. Section 5 distinguishes two ideas that get packaged together in Williamson's world view: one idea is that existence at any type does not come and go from world to world (this idea is naturally articulated using a suitably typed version of the Barcan formula). The second idea is that existence comes cheap so that, for example, whenever we have a meaningful sentence there is an associated proposition. This is naturally articulated using a suitably typed comprehension principle. The two ideas are natural allies for Williamson, since both the Barcan formulae and comprehension can be derived from universal instantiation with minimal additional assumption, however the free logician who rejects UI is free to take a very different view of these two strands of Williamson's world-view. In section 6 we raise a variety of problems free logical packages. In section 7 we articulate and critically assess different styles of ramificationist approaches - including one that merely ramifies quantifiers, one that merely ramifies operators and another that ramifies both. In this connection we introduce a second kind of puzzle due Saul Kripke. Having that puzzle, as well as our focal puzzle, in view allows us to better assess the merits of the various styles of ramification.

\section{Priorean Paradoxes and Free Quantificational Logic}

As we saw above, non-redundant stratification involves dropping the unrestricted principle of universal instantiation. Indeed, as we shall see, it is the rejection of UI that is the key to blocking certain of the intensional paradoxes, whether or not that is motivated or accompanied by a stratificational picture. This is because UI is all by itself sufficient for deriving certain paradoxes that can't be derived without UI. Here we have in a mind a family of paradoxes associated with Prior and Kaplan.

Let's begin by discussing a puzzling result of Prior 1961 [19]. Here Prior is working in a small fragment of the Fregean hierarchy, consisting of nothing but the propositional calculus augmented with a device for quantifying into the position of sentences, and a unary sentential operator, $Q$, whose interpretation can be left completely open for the purposes of this result. ${ }^{9}$ Armed with a pair of assumptions, namely UI for the quantifiers into sentence position, and classical propositional logic, one can prove the following theorem. ${ }^{10}$

Prior's Theorem: $Q \forall p(Q p \rightarrow \neg p) \rightarrow \exists p(Q p \wedge p) \wedge \exists p(Q p \wedge \neg p)$.

To get a feel for why this theorem is so puzzling, let us look at a particular interpretation for $Q$ by reading $Q p$ as 'Kaplan believes that $p$ '. So interpreted, Prior's theorem says roughly ${ }^{11}$ that if Kaplan believes that everything that he believes is false, then he believes something true and also believes something false.

As it happens, the material conditional in question is almost certainly true, for mundane reasons - for example, we doubt very much that Kaplan believes that everything he believes is false, or that he believes only true or only false things. Nevertheless, it is extremely surprising

\footnotetext{
${ }^{9}$ Whereas Prior's original discussion formulated in Polish notation and used the symbol $d$ for the relevant unary operator, Kaplan used more standard notation and used the symbol $Q$. For the sake of typographical uniformity we shall use $Q$ throughout. Had Prior not been using Polish notaton, we suspect his paper would have entered the philosophical canon much earlier.

${ }^{10} \mathrm{~A}$ complete axiomatisation of the the logic of quantification into sentence position would include several other axioms governing the propositional quantifiers, however this argument only requires UI, at least given certain non-trivial decisions about which truth functional connectives to take as primitive.

${ }^{11}$ We say 'roughly' because we are using the English 'if then' to interpret the material conditional, we are using singular quantification instead of quantification that takes sentence position, and finally we are employing truth and falsity predicates that make no appearance in Prior's actual theorem.
} 
to be informed that this fact has the status of a logical truth. This doesn't seem like the sort of thing that could be figured out from logic alone.

One could learn to live with the necessary truth of this instance of Prior's theorem, maintaining perhaps that belief is closed under logical consequence, so that one can't believe anything false uniquely. It's not clear that these speculations about belief would render the relevant instance of Prior's theorem a logical truth. But more importantly, it doesn't speak to the full generality of the theorem. Other interpretations of $Q$ are harder to live with. Consider, for example:

1. If Kaplan says at midnight that everything Kaplan says at midnight is false, then Kaplan has said a true and a false thing at midnight.

2. If at midnight Kaplan writes on a whiteboard that everything Kaplan writes on a whiteboard at midnight is false, then Kaplan writes on a whiteboard a true and a false thing at midnight.

3. If Kaplan seems to have said at midnight that everything Kaplan seems to have said at midnight is false, then there is a a true and a false thing Kaplan seems to have said at midnight.

4. If at midnight it seems that Kaplan writes on a whiteboard that everything at midnight it seems that Kaplan writes on a whiteboard is false, then there's something true that at midnight it seems that Kaplan is writing on a whiteboard, and there's something false that at midnight it seems that Kaplan is writing on a whiteboard.

Prior attempted to explain the truth of 1 and 2 in the face of apparent counterexamples by suggesting that even though certain speeches or sentences seem to say something, they in fact fail to do so. Suppose for example that Kaplan had at midnight written nothing but the sentence 'everything Kaplan writes on a whiteboard at midnight is false' on the whiteboard. Although it seems as though Kaplan has written that everything he wrote on the whiteboard at midnight is false, Prior suggests that what seems to be so isn't so, and indeed, that the inscription on the whiteboard in this case has failed to express anything. ${ }^{12}$

As Prior was aware, his response was far from pain free. However the strategy just outlined is particularly unsatisfying as an explanation of the truth of 3 and 4. Applying that strategy to these cases involves denying that it even seems that Kaplan writes that everything that Kaplan seems to write at midnight is false. ${ }^{13}$

While we may in the end have to grudgingly accepting these results, we think that their strangeness provides excellent motivation for thoroughly exploring the alternatives. In this instance the alternatives are very clear cut: short of relinquishing propositional logic, the only other principle appealed to in the proof of Prior's theorem was the principle of universal instantiation. $^{14}$

\footnotetext{
${ }^{12}$ Obviously this isn't the only way to reconcile Prior's theorem with the apparent data. One could also insist that despite appearances Kaplan wrote two or more things at midnight (see, for example, Bradwardine [3], Dorr [7], Slater [23]). Or one might insist that while Kaplan didn't say what he seemed to say, he succeeded in saying something else Smith [24], Bacon [1]. The challenge for these kinds of responses is to offer some guidance as to what the additional or alternative contents are.

${ }^{13} \mathrm{~A}$ similar worry arises for the alternative and multiple proposition views. The alternative proposition view has to deny what seems obvious, namely the antecedent of 4 , while the multiple proposition view must deny something else that seems obvious, namely the negation of the consequent of 4 .

${ }^{14}$ We should mention in passing another kind of paradox where denying universal instantiation might provide a path to a distinctive kind of solution. Here is a principle about propositional identity that is prima facie
} 
Let us now show how we can give a distinctive diagnosis of 1-4 in a setting where universal instantiation is relaxed. Recall that Prior's account of 2 is that while Kaplan wrote down the sentence 'everything Kaplan writes on a whiteboard at midnight is false' he (i) didn't write at midnight that everything Kaplan writes on a whiteboard at midnight is false, and indeed (ii) he didn't write anything whatsoever at midnight. The free logical approach we're exploring can accept (ii) without thereby accepting (i). For once universal instantiation is relinquished, the inference from Kaplan didn't write anything at midnight to Kaplan didn't write that everything Kaplan writes on a whiteboard at midnight is false is invalid. Similarly, in the case of 4, the inference from 'there's nothing that Kaplan seemed to write at midnight' to 'it's not the case at midnight that Kaplan seemed to write that everything Kaplan writes on a whiteboard at midnight is false' is invalid as well.

The approach here shares much with a similar free logical approach to the problem of empty names in the first-order context. In that setting the free logician can maintain that Alice said that Pegasus flies without thereby committing us to inferring that there's something that Alice said flies. ${ }^{15}$

Note that even if Kaplan didn't in fact uniquely write 'everything Kaplan writes on a whiteboard is false' on a whiteboard, it's clear that he could have done so. The free logical approach described above will accommodate the idea that Kaplan could have written that everything Kaplan writes on a whiteboard is false without there being both a true and a false thing written. Let us abbreviate $\forall p(Q p \rightarrow \neg p)$ as $\lambda$. Then this amounts to:

$$
\diamond(Q \lambda \wedge \forall q(Q q \rightarrow(q \leftrightarrow \lambda)))
$$

But this, notice, conflicts with the necessity of Prior's theorem: $\square(Q \forall p(Q p \rightarrow \neg p) \rightarrow \exists p(Q p \wedge$ $p) \wedge \exists p(Q p \wedge \neg p))$. The necessity of Prior's theorem is derivable if we assume that UI is necessary, and that the laws of propositional logic preserve necessity. ${ }^{16}$ The free logical approach accommodates $\mathrm{K} 1$ by relaxing universal instantiation. Note that this principle is not even prima facie plausible unless $Q$ is replaced by certain sorts of attitudes - it is not plausible if we replace $Q$ for 'it is true that', or even 'it is necessary that' or 'it is known that'.

The motivation for K1 seems to generalize to any sentence of English. Roughly speaking, it seems that for any meaningful sentence ' $\phi$ ' of English Kaplan could have written ' $\phi$ ' on the whiteboard at midnight and nothing else. By a completely analogous thought, it seems that we ought to be able to infer that Kaplan wrote that $\phi .{ }^{17}$ Thus if we are sympathetic to K1 for

compelling, and particularly compelling if you think of propositions in a structured way: if $Q$ and $Q^{\prime}$ are propositional operators with different extensions, then the proposition that something is $Q$ and the proposition that something is $Q^{\prime}$ are not identical (more formally: $\left(\exists p Q p \equiv \exists p Q^{\prime} p\right) \rightarrow \forall p\left(Q p \leftrightarrow Q^{\prime} p\right)$ ). But now a higherorder analogue of the Russell-Myhill paradox threatens to show that this is inconsistent. The derivation of this essentially involves the principle of universal instantiation into sentential position (Uzquiano [29]). It is beyond the scope of this paper to explore the promise of this response. However we should note that the models in this paper are generally not useful for modelling structured propositions, since, while they allow modally equivalent propositions to be distinct, they treat logically equivalent propositions as identical.

${ }^{15}$ Both the first order free logician described above, and the higher-order free logician will therefore have to be positive free logicians. That is, they will reject the inference of $\exists x \phi$ from $\phi[t / x]$ even for atomic instances of $\phi$.

${ }^{16}$ One could also prove this by directly appealing to a general rule of necessitation, however this rule is not uncontroversial in this context as it is relinquished, for example, in Kaplan's logic of demonstratives in [10], and the status of necessitation in Williamson's book is complicated. The above proof does not rely on necessitation - in general most appeals to necessitation can be eliminated in favour of slightly stronger (necessitated) premises.

${ }^{17}$ We are, of course, taking a few liberties here: for example, if the report is made after the writing then we would use different tense morphology. 
these kinds of reasons one should be strongly tempted to accept a schema that generalises K1:

$$
\diamond(Q \phi \wedge \forall q(Q q \rightarrow(q \leftrightarrow \phi)))
$$

where $\phi$ is any closed sentence.

$\mathrm{K} 1$ is just the instance of $\mathrm{K} 2$ you get when you substitute $\phi$ for $\forall p(Q p \rightarrow \neg p)$.

The principle K2 is closely related to, but subtly different from a thesis that is like K2 but which universally generalises into sentence position.

$$
\forall p \diamond(Q p \wedge \forall q(Q q \rightarrow(q \leftrightarrow p)))
$$

$\mathrm{K} 3$ initially looks as though it is straightforwardly stronger than $\mathrm{K} 2$, since it talks about all propositions, not just those expressible in a particular language. However to get K2 from K3 one must employ an instance of universal instantiation (instantiating $p$ with $\phi$ ) which is not available in the free logical setting that we're currently exploring. Indeed, for the same reason $\mathrm{K} 1$ doesn't follow from K3, for that would require instantiating $p$ with $\lambda$ (i.e. the non-existent proposition that everything Kaplan says is false).

$\mathrm{K} 3$ is itself a straightforward consequence of a thesis sympathetically discussed by Kaplan which replaces the final material equivalence with an identity connective. ${ }^{18}$

$$
\forall p \diamond(Q p \wedge \forall q(Q q \rightarrow(q=p)))
$$

We may note in passing that there are principles that strengthen K1 and K2 in a way analogous to the way that $\mathrm{K} 3^{=}$strengthens $\mathrm{K} 3$. The difference will not matter much in what follows - the models that we consider later will also validate these strengthened principles.

Discussions of $\mathrm{K}{ }^{=}$, beginning with Kaplan's own discussion, often focus on putative explanations of the falsity of that principle that turn on cardinality considerations (e.g. Lewis [13], Davies [5], Divers [6]). ${ }^{19}$ The key line of thought is that there are more collections of possible worlds than there are possible worlds - it can't be that for every collection of worlds there is a world at which the proposition corresponding to that collection is uniquely thought. ${ }^{20}$ (Kaplan's own discussion focuses on cardinality arguments for the logical falsity of $\mathrm{K} 3^{=}$. Kaplan was happy with the thought that $\mathrm{K} 3^{=}$was false, but unhappy with the thought that it was false as a matter of logic; as he saw things standard possible worlds semantics delivers the latter unwanted conclusion and concluded that this semantics is thereby unsatisfactory. We will return to these issues in section 7.1.) However cardinality considerations have no direct application to the puzzles we obtained from K1-K3. For unlike K3 ${ }^{=}$, for example, K3 doesn't require different worlds to serve as witnesses for propositions with different modal profiles: one and the same possible world could be a witness for the possibility that a certain proposition is thought, and simultaneously a witness for the the possibility of thinking a different proposition so long as that proposition has the same truth value of the former at that world (regardless of whether it has the same truth value at other worlds). Moreover, in the case of K1 and K2, there is an even simpler reason why cardinality considerations are beside the point: K2 is a schema with countably many instances, and there are certainly enough possible worlds to witness each

\footnotetext{
${ }^{18}$ Kaplan's sympathy is not directed at the truth of this sentence, but at its logical coherence.

${ }^{19}$ It should be noted that most of these authors, while attributing the paradox to Kaplan, did not have access to the details of Kaplan's text since it had not been published.

${ }^{20}$ Of course if you're a hyperintensionalist there won't be any such thing as the proposition corresponding to a set of worlds. But if there are more propositions than collections of possible worlds, that only makes the situation worse for the principle.
} 
instance. Moreover, $\mathrm{K} 1$ concerns only one proposition, $\lambda$ ! Note, thus, that even $\mathrm{K} 1^{=}$and $\mathrm{K} 2^{=}$ are similarly immune to cardinality worries.

\section{Higher-order Necessitism and Higher-Order Free Logic}

Our principle of universal instantiation is also at the center of the higher-order necessitist/contingentist debate; in this case, as it concerns quantification into sentence position. Given necessitation and the principle UI one can quickly derive the central theses of higher-order necessitism. For quantification into sentence position this amounts to:

$$
\square \forall p \square \exists q p \equiv q
$$

Here the symbol $\equiv$ is a connective expressing the analogue of identity at the type of sentences. However we shall leave it open whether this notion can simply be defined in terms of necessary equivalence, where $p \equiv q$ is defined as $\square(p \leftrightarrow q)$, or perhaps in terms of higher-order quantification into operator position by analogy with Leibniz's law, so that $p \equiv q$ is defined as $\forall Q(Q p \rightarrow Q q) \cdot{ }^{21}$

Recall that universal instantiation is equivalent to the the principle of existential introduction. One proof of NNE - that relies on the principle of necessitation - starts with the theorem $p \equiv p,{ }^{22}$ and infers $\exists q p \equiv q$ by existential introduction. By necessitation, which allows one to prefix any theorem with a box, one infers $\square \exists q p \equiv q$. By universal generalization, we get $\forall p \square \exists q p \equiv q$, and finally by necessitation again we get $\square \forall p \square \exists q p \equiv q$. Instead of relying on a general principle of necessitation, one could start instead with a suitably necessitated version of UI as a premise. This is closer to Williamson's own argumentative path as he is reluctant to rely on the principle of necessitation. ${ }^{23}$

The first-order analogue of NNE - where $\equiv$ is interpreted as first-order identity - is often discussed in connection with the Barcan formula (a first-order analogue of BF below) and its converse (an analogue of CBF).

$$
\begin{aligned}
& \forall p \square A \rightarrow \square \forall p A \\
& \square \forall p A \rightarrow \forall p \square A
\end{aligned}
$$

Just as in the first-order setting, NNE is a consequence of CBF which can be seen by taking $A$ to be $\exists q p \equiv q$.

Like NNE, CBF is also a consequence of UI, given some weak standard background assumptions about the logic of necessity. BF is not forced on us by the principles we have considered so far, but natural assumptions about the logic of necessity guarantee it. Here we have in mind the Brouwer axiom: $p \rightarrow \square \diamond p .^{24}$

\footnotetext{
${ }^{21}$ Given universal instantiation the latter definition satisfies schematic Leibniz's law, but without it we cannot derive it (we can derive quantified LL either way); thus the latter definition only seems appropriate assuming UI.

${ }^{22}$ This is a theorem under either definition of $\equiv$, as it amounts to $\square(p \leftrightarrow p)$ and $\forall Q(Q p \rightarrow Q p)$ respectively.

${ }^{23}$ To be more precise, Williamson relies on a suitably necessitated version of a consequence of UI, namely, Comp. See below.

${ }^{24}$ The proofs of these are well known (see Prior [18]). In fact much weaker axioms suffice for this purpose; for example the principle $\square(p \rightarrow \square p) \rightarrow(\diamond p \rightarrow p)$ (see Bacon [2]).
} 
Another principle that plays a pivotal role in Williamson's book is a principle of comprehension for each type. For quantification into sentence position, it amounts to: ${ }^{25}$

$$
\square \exists p \square(p \leftrightarrow \phi)
$$

One might think that Comp can be derived from NNE alone. Indeed if we understand $\equiv$ as necessary equivalence, then Comp simply becomes $\square \exists p p \equiv \phi$ which looks like it just follows from an instance of NNE. However this latter inference - from $\square \forall q \square \exists p p \equiv q$ to $\square \exists p p \equiv \phi-$ relies on UI. The relationship between NNE and Comp is much like that between K3 and K2: without the help of UI, one can't prove the instance from the universally generalised claim (we will see models which demonstrate this presently).

Since UI entails all of the central theses associated with higher-order necessitism, it's clear that if one wants to be a higher-order contingentist in any reasonable sense then one has to reject UI.

It's worth noting that although the central theses of necessitism listed above do not themselves require UI, the rule fits well with a wider package of views that Williamson holds. ${ }^{26}$ In fact, this wider package plays an important role in Williamson's abductive argument for necessitism: as Williamson notes in [32] the result of combining standard quantificational principles (including UI) and standard modal principles has necessitism as a consequence. ${ }^{27}$ Thus although, as we shall presently see, one can be a necessitist without accepting UI, this would undercut one of the primary motivations for being a necessitist. Thus insofar as one responds to Prior's paradox by adopting a free logic that relaxes UI, one has less reason to be a necessitist.

\section{How to Model K1, K2 and K3}

In what follows we shall be looking at illustrative models of the language fragment of interest: quantified propositional logic (with propositional constants $T, \perp$ ), supplemented with a modal operator, $\square$, and a sentential operator, $Q$, and a propositional identity connective $\equiv$. We will be primarily concerned with Kripke-style models of this language: these consist of (i) a set of points, $W$, (ii) a domain for the propositional quantifiers at each point $w \in W$, consisting of

\footnotetext{
${ }^{25}$ Notice that in Williamson's system there is a subtle distinction between comprehension principles for quantification into sentence position, and quantification into, say, monadic predicate position. The first is derivable from UI, but derivation of the second from UI requires additional assumptions about what counts as a predicate. In Williamson's system the only monadic predicates are simple monadic predicates (whereas it's obviously not true that all sentences are simple), or at any rate he doesn't explicitly license a device for converting any old open sentence into a monadic predicate (such as $\lambda$-abstraction). Absent such a device one cannot, for example, derive $\exists F \square \forall x(F x \leftrightarrow(G x \wedge H x))$. And this is essentially because in order to use existential quantifier introduction to move from $\square(G x \wedge H x \leftrightarrow G x \wedge H x)$ to $\exists F \square \forall x(F x \leftrightarrow G x \wedge H x)$ one would need to first convert the open sentence $G x \wedge H x$ into a type suitable for monadic second-order existential generalization. Given that the focus of this paper is on propositional necessitism, these subtleties are not very relevant to the main discussion.

${ }^{26}$ That NNE, BF and CBF do not entail UI will be demonstrated by the models we consider in section 4 . To see that Comp does not entail UI, we must consider a model in which propositions are not individuated by necessary equivalence. For example, in the model $W=\{x, y\}, R=\{\langle x, x\rangle,\langle y, y\rangle\}, D(w)=\{\{w\}, \emptyset\}$,

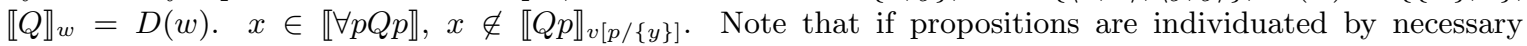
equivalence then UI can be proven from Comp.

${ }^{27}$ In [34] Williamson focuses instead on abductive arguments for Comp which in turn guarantee many of the necessitist theses. There Williamson describes a sound (but incomplete) axiomatisation of higher order modal logic. In that system Comp is not an axiom, but a theorem derived in the above way from UI. This shift of focus from UI to Comp perhaps seems reasonable given his simplifying assumption that higher-order entities are individuated by necessary equivalence.
} 
a set of sets of points $D(w) \subseteq \mathcal{P}(W)$, (iii) an accessibility relation $R \subseteq W \times W$ to interpret $\square$, and (iv) an extension for $Q$ at each point, which can be represent by a function, written $|Q|$, which maps each point to a set of sets of points. In what follows we shall be concerned exclusively with models that validate $\mathrm{S} 5$, in which $R$ is an equivalence relation.

In what follows we shall adopt a sharp distinction between arbitrary sets of points, which we shall call an intension, and a set of points that that belongs to the domain, $D(w)$, of some world $w$ - the latter kind of set corresponds to a proposition at that world: an intension that is in the range of the propositional quantifiers there.

$|Q|$ maps each point to the set of intensions that are $Q$ at that point, where an intension is given by any set of points. As noted $D(w)$ is a set of intensions in this sense representing the propositions that exist at $w$. Note, however, that the extension of $Q$ at $w$ might include intensions that don't exist at $w$. Note also that we have not insisted that the intensions that exist or that are $Q$ at $w$ consist only of sets of points accessible to $w$. In certain places we will consider hyperintensional models in which distinct intensions are necessarily equivalent, where such a constraint would be inappropriate (in these models an arbitrary set of points might be more appropriately called a 'hyperintension', although we'll stick to our terminology for consistency). In these kinds of models two intensions may agree at every accessible point, but nevertheless be represented by different sets of points; the difference may show up when these propositions are embedded under $Q$ and $\equiv$ even when they don't show up when embedded under $\square .^{28}$

To complete the specification of the model we need the notion of a variable assigment: a function, $v$, from propositional variables to sets of points. A set of points can now be assigned recursively to every sentence of our language (relative to an assignment) in the following way: ${ }^{29}$

$$
\begin{aligned}
& \llbracket P \rrbracket_{v}=v(P) \text { if } P \text { is a propositional variable and } \llbracket P \rrbracket \text { if an atomic sentence letter. } \\
& \llbracket \phi \equiv \psi \rrbracket_{v}=W \text { if } \llbracket \phi \rrbracket_{v}=\llbracket \psi \rrbracket_{v} \text { and }=\emptyset \text { otherwise. } \\
& \llbracket \phi \vee \psi \rrbracket_{v}=\llbracket \phi \rrbracket_{v} \cup \llbracket \psi \rrbracket_{v} \\
& \llbracket \neg \phi \rrbracket_{v}=W \backslash \llbracket \phi \rrbracket_{v} \\
& \llbracket \square \phi \rrbracket_{v}=\left\{w \in W \mid R(w) \subseteq \llbracket \phi \rrbracket_{v}\right\} \\
& \llbracket Q \phi \rrbracket_{v}=\left\{w \in W\left|\llbracket \phi \rrbracket_{v} \in\right| Q \mid(w)\right\} \\
& \llbracket \forall p \phi \rrbracket_{v}=\left\{w \in W \mid w \in \llbracket \phi \rrbracket_{u}, \forall u \text { such that } u[p] v \text { and } u(p) \in D(w)\right\}
\end{aligned}
$$

Here we have used $R(w)$ to denotes the set of worlds accessible to $w(\{x \mid R w x\})$ and $u[p] v$ to mean that $v$ and $u$ agree except, possibly, on which set of points they have assigned to $p$.

As in the case of first-order free logic, the fact that the domain of the quantifiers is non-empty does not come for free, although one can ensure it by requiring models to satisfy all instances of the schema $\forall x \phi \rightarrow \exists x \phi$. Generalising to the propositional case, we want the principle $\forall p \phi \rightarrow \exists p \phi$ to be satisfied in our models, which is guaranteed by requiring that $D(w) \neq \emptyset$ for each $w \in W$. In what follows we shall refer to models of this type as 'regular models'. Regular models are models of propositionally quantified S5, with a free logic for the quantifiers and the

\footnotetext{
${ }^{28}$ It should be noted that it would also be inappropriate to restrict attention to universal accessibility relations - as is often done when, like us, one is dealing with models of S5 - but in the current context that is unwise since we wish to allow models in which $Q$ is hyperintensional.

${ }^{29} \mathrm{~A}$ richer language might also include propositional letters, each of which denotes a set of points under an interpretation, but we will have no need for them here.
} 
principle $\forall p \phi \rightarrow \exists p \phi$; thus the existence of a regular model of some principle guarantees the consistency of that principle with this fragment of propositionally quantified modal logic. If the domain of propositions is empty at some world we shall refer to the model as 'non-regular'.

It should be noted that according one prominent tradition, the intended model of a higherorder language ought itself be specified in a higher-order metalanguage (Tarski [25] §4, Rayo and Uzquiano [28], Rayo and Williamson [30], Williamson [33]). ${ }^{30}$ Similarly one might also insist that the intended model of a modal language itself be described in a modal metalanguage. Thus, for example, Prior and Fine (see the appendix of [20]) replace first-order quantification over times and worlds with quantification into sentence position, and simulate worlds and times by (the higher-order equivalent of) talk of propositions that are only ever true at most once, or propositions that are possibly both true and necessitate every truth.

Our model theory above is presented in the first-order language of set theory, and so does not have this form. However, our purpose is not to specify the intended model of $\mathcal{L}$ but to figure out which collections of principles are consistent with one another. Since consistency is a mathematical consequence of the existence of any kind of model, a Kripke-style model theory will suffice for our purposes.

\subsection{Finite Models}

Note that models of K1-K3 are easy to come by on their own. The most flat-footed way to construct a model of these principles is to consider models of a higher-order form of nominalism: suppose that universal quantification into sentence position always yields vacuous truth, because, loosely speaking, there are no such things as propositions. ${ }^{31}$ Then K3 is vacuously true because it begins with a universal quantifier. As for $\mathrm{K} 1$ and $\mathrm{K} 2$, one can ensure their truth by having a possible world where $Q \phi$ is true for any interpretation of $\phi$ - this could be achieved simply by letting the extension of $Q$ at this world include every set of worlds. So for example, $W=\{w\}, R=\{\langle w, w\rangle\}, D(w)=\emptyset,|Q|(w)=\{\emptyset,\{w\}\}$. (Recall that $D(w)$ is not the domain of accessible worlds, it is the domain of existing propositions - in this case no propositions exist at $w$.)

Thus we get the following proposition:

Proposition 4.1. K1, K2, and K3 have a finite constant domain non-regular model.

Note that because this is a non-regular model, we do not get the principle $\forall p \phi \rightarrow \exists p \phi$ which requires the domain to be non-empty. Indeed, one might want to go further and require not only that the propositional domains be non-empty but that they contain both $T$ and $\perp$. Indeed, if the domain contains only these two propositions we also get the desirable thesis that the existing propositions are closed under arbitrary Boolean operations.

With this mind, let us consider a slightly more interesting model of K3. Here we let there be two worlds $W=\{x, y\}$ with a universal accessibility relation, such that at both worlds the necessary and impossible propositions are the only propositions that exist: $D(x)=D(y)=$ $\{\{x, y\}, \emptyset\}$. Since there are only two existing propositions at either world, it is easy to find an interpretation of $Q$ in which each of those propositions is the only proposition that is $Q$

\footnotetext{
${ }^{30}$ For example, one might take Frege's 'concept horse' problem to be a result of not properly acknowledging this idea; see Williamson [33] for this and other reasons for dissatisfaction with first-order models.

${ }^{31}$ Note that this is a model of higher-order nominalism, and moreover we don't use higher-order quantification in the articulation of the models. However, the model itself is of course not nominalistically acceptable in a broader sense, because the models are constructed out of abstract objects, and so admit first-order objects that nominalists have historically rejected.
} 
at some world: $|Q|(x)=\{\{x, y\}\}$ and $|Q|(y)=\{\emptyset\}$. Thus K3 is validated at both worlds in this model. It should be noted that the propositions that exist at each world satisfy a number of closure conditions: propositions corresponding to $\top$ and $\perp$ exist, and an arbitrary Boolean combination of existing propositions exists. However existence is not closed under the $Q$ operator. For example $Q \top$ is true at the set of worlds $\{x\}$, but $\{x\}$ does not exist even though the set of worlds $T$ corresponds to does.

What about K1 and K2? The set of worlds at which Prior's sentence, $\lambda$, is true is $\{y\}$ : at $x$ a truth is $Q$, whereas at $y$ only the inconsistent proposition is $Q$. Since $\{y\}$ is not $Q$ at any world, $Q \lambda$ is not true at any world, and so $\diamond Q \lambda$ is also false at both worlds. This precludes K1 being true, and thus also the schema K2 of which $\mathrm{K} 1$ is an instance.

We could validate $\mathrm{K} 1$ by changing our model slightly. Let $W=\{x, y, z\}$, and as before we stipulate that $R$ is universal and moreover that only $W$ and $\emptyset$ exist at each world, and that $|Q|(x)=\{W\}$ and $|Q|(y)=\{\emptyset\}$. Finally we stipulate that $|Q|(z)=\{\{y, z\}\}$. Notice that K3 is valid in this model for the same reason as before. In this model $\lambda$ is true at $y$ and $z$ (it's true at $y$ because only $\perp$ is $Q$ at $y$, and vacuously true at $z$ because nothing that exists is $Q$ at $z$ ). Thus $\lambda$ expresses $\{y, z\}$, and thus $Q \lambda$ is true at $z$. Moreover $\forall p(Q p \rightarrow \lambda \equiv p)$ is vacuously satisfied at $z$, so the principle $\mathrm{K} 1-\diamond(Q \lambda \wedge \forall p(Q p \rightarrow \lambda \equiv p)$ - is true at every world. Notice however that we can still find instances of K2 that are not validated. For example $\neg \lambda$ has the singleton $\{x\}$ as its intension, which is not in the extension of $Q$ at any world, so the $\neg \lambda$ instance of K2 is false at every world. Note as before that existence is closed under arbitrary Boolean operations, but not under the $Q$ operator.

Let us see if we can push this strategy further and validate all three $\mathrm{K}$ principles. So far we have only considered models in which one set of worlds appears in extension of $Q$ at each world. But if we drop that constraint we can generate a three world model that validates K1-K3. Let $W=\{x, y, z\}$, and let the extension of $Q$ at $x$ and $y$, the accessibility relation, and the existing propositions, be as before. Now let the extension of $Q$ at $z$ be $\mathcal{P} W \backslash\{W, \emptyset\}$. K3 is validated as above. K2 is validated because for any sentence $\phi$ that is true at some but not all worlds is such that (i) its intension is $Q$ at $z$ and (ii) it is vacuously true that every existing proposition at $z$ that is $Q$ is equivalent to it. Meanwhile, if $\phi$ is true at every world or none, then its extension will be $Q$ without any existing proposition being $Q$ at $x$ and $y$ respectively.

Proposition 4.2. K1, K2, and K3 have a finite constant domain regular model containing $\top$ and $\perp$ and closed under arbitrary Boolean operations.

This model is peculiar: it secures the validity of $\mathrm{K} 2$ by having the extension of $Q$ be radically non-unique at $z$, even though the object language statement of uniqueness is satisfied at $z$. For example at $z, \lambda$ (i.e. the set of points $\{y, z\})$ is $Q$ without any existing proposition being $Q$, and, likewise, $\neg \lambda$ (i.e. the set of points $\{x\}$ ) is $Q$ without any existing proposition being $Q$

The sense in which $\lambda$ and its negation are uniquely $Q$ is by no existing proposition being $Q$ alongside them. What we want is to be able to impose a stronger uniqueness requirement, however this turns out to be hard to express in the free logical setting. By analogy suppose we wanted to say that someone is imagining Pegasus but not Atlantis or Zeus or .... The '...' cannot be captured quantificationally, because a quantificational claim won't entail the instances involving fictional names. ${ }^{32}$ However we could introduce an operator $Q^{*} \phi$ for this purpose. The

\footnotetext{
${ }^{32}$ One could attempt to state this using substitutional quantification, but this may have limitations. Suppose the object language lacked Meinongian quantifiers, but the theorist had them. Then the theorist could express the shortcomings by pointing out that there may be fictional things in the broad sense for which there were no names, and that imagining one of those alongside Pegasus would a counterexample to the intended meaning of the elliptical claim. Exactly the same point applies if one attempts to use substitutional quantification to
} 
interpretation of ' $Q *$ snow is white' is that it mean the same as 'snow is white is $Q$ but grass is green is not $Q$, nor ...' where the ellipsis is understood in this open ended way. Two schemata partially characterising the behaviour of $Q^{*}$ can be outlined:

$$
\begin{aligned}
& Q^{*} \phi \rightarrow Q \phi \\
& Q^{*} \phi \wedge Q^{*} \psi \rightarrow \phi \equiv \psi
\end{aligned}
$$

These schemata must be understood open endedly. ${ }^{33}$ Model theoretically, we can interpret $Q^{*}$ in our models as follows:

$$
\left|Q^{*}\right|(x)=\emptyset \text { if }|Q|(x) \text { is not a singleton, and }=|Q|(x) \text { otherwise. }
$$

Armed with $Q^{*}$ there's a sort of uniqueness demand that strengthens K1-K3 that can be articulated as follows:

$$
\begin{gathered}
\diamond Q^{*} \lambda \\
\diamond Q^{*} \phi \\
\forall p \diamond Q^{*} p
\end{gathered}
$$

However $\mathrm{K}^{+}$fails in our present model, for example $\diamond Q^{*} \lambda$ is not true in the present three world model. In fact, no three world model will do. ${ }^{34}$ This idea generalises to show that no finite world model will do: there can be no finite model in which the set of worlds expressed by each sentence is the one and only member of $|Q|(w)$ for some world $w$ (and thus no finite model of $\mathrm{K}^{+}$). This holds even when restricting attention to the original language without $Q^{*}$.

Proposition 4.3. If $\langle W, D, R,|Q|\rangle$ is a model such that for each closed sentence $\phi$, there is a point $w \in W$ such that $|Q|(w)=\{\llbracket \phi \rrbracket\}$, then $W$ must be an infinite set of points.

Proof. We shall show that in any such model, for any $n<m, \llbracket Q^{n} \top \rrbracket \neq \llbracket Q^{m} \top \rrbracket$ (this precludes the satisfaction of our condition in a finite model, because there are infinitely many sets of worlds expressed by sentences in the language). Suppose that this hypothesis holds for every $n<m$. Suppose for contradiction that for some $n<m+1, \llbracket Q^{n} \top \rrbracket=\llbracket Q^{m+1} \top \rrbracket . n$ cannot be 0 since this entails that $\llbracket Q^{m+1} \top \rrbracket=$ $\llbracket \top \rrbracket=W$ so that $\llbracket Q^{m} \top \rrbracket$ is $Q$ at every world. Since $\llbracket Q^{m} \top \rrbracket$ can’t be identical to both $W$ and $\emptyset$, either there's no world where $W$ is the only member of the extension of $Q$ or there's no world where $\emptyset$ is the only member of the extension of $Q$. So $n>0$, and $\llbracket Q^{n} \top \rrbracket=\llbracket Q^{m+1} \top \rrbracket$, which means that for every world $w$ such that $\llbracket Q^{n-1} \top \rrbracket \in|Q|(w), \llbracket Q^{m} \top \rrbracket \in|Q|(w)$. By inductive hypothesis $\llbracket Q^{n-1} \top \rrbracket \neq \llbracket Q^{m} \top \rrbracket$, so there's no world where $\llbracket Q^{n-1} \top \rrbracket$ is the unique member of the extension of $Q$.

\footnotetext{
capture the claim 'I'm thinking that snow is white, but I'm not thinking that grass is green or ...'

${ }^{33}$ See [15]. Roughly, a schema is understood open endedly if it holds under any extension of the language.

${ }^{34}$ To satisfy $\mathrm{K}^{+}$then there must be two distinct worlds that make $Q^{*} \top$ and $Q^{*} \perp$ true respectively. $Q^{*} \top$ and $Q^{*} \perp$ are therefore both contingent and different. $\top, \perp, Q^{*} \top$ and $Q^{*} \perp$ require four different worlds as witness to the relevant instances of $\mathrm{K}^{+}$, but there are only three worlds to go around.
} 


\subsection{Infinite Models}

It's natural to wonder if there are any infinite models satisfying the constraint. We can construct one in the spirit of the earlier models - involving a fairly frugal domain of propositions - as follows. Let $W=\mathbb{N}$, let the accessibility relation be universal, and as before let $D(n)=\{W, \emptyset\}$. Now pick some bijection, $\sigma$, between $\mathbb{N}$ and the set of finite and cofinite subsets of $\mathbb{N} .{ }^{35}$ Given $\sigma$ the extension of $Q$ can specified as: $|Q|(n)=\{\sigma(n)\} .{ }^{36}$ In this model every finite and cofinite set is the unique member of the extension of $Q$ at exactly one world. Moreover, it can be shown by a simple induction that in this particular construction every closed sentence of our language expresses a finite or cofinite set. ${ }^{37}$ Thus it follows that in this model, for every set of worlds expressed by some sentence, there is a world where that set is the unique member of the extension of $Q$. So we have a model of $\mathrm{K} 1-\mathrm{K} 3$, and also a model of $\mathrm{K}^{+}-$and $\mathrm{K}^{+}$and $\mathrm{K}^{+}$. Just like in the earlier models, the propositions that exist at each world are closed under all Boolean operations (including infinitary conjunction and disjunction), but are not closed under the $Q$ operator. For example, the set corresponding to $\top$ exists, but $Q \top$ is true at exactly one world, and no singleton set is in the domain of quantification at any world.

Proposition 4.4. $\mathrm{K}^{+}, \mathrm{K} 2^{+}$, and $\mathrm{K} 3^{+}$have an infinite constant domain regular model whose domain contains $T$ and $\perp$ and is closed under arbitrary Boolean operations.

The models considered so far might be deemed uninteresting because they only permit quantification over the necessary and impossible proposition: there is both a paucity of propositions (there are only two) and a lack of variety (there are no contingent propositions). It's easy to modify the model so that it allows a few more propositions: for example, we could take a finite number of finite sets, and close them under Boolean operations, and the resulting model would still satisfy $\mathrm{K}^{+}{ }_{-}-\mathrm{K} 3^{+}$. Of course, this model will only deliver finitely many existing propositions in total since closing finitely many finite sets under Boolean operations will only yield finitely many sets. As a corollary, existing propositions will not be closed under $Q$ in these models: if $\top$ expresses an existing proposition, and existence was closed under $Q$ then, $Q^{n} T$ would express an existing proposition for each $n$. By the result above, any model of this sort that satisfies $\mathrm{K}^{+}$or $\mathrm{K}^{+}{ }^{+}$will be such that $Q^{n} \top$ expresses a different proposition for each $n$, so there must be infinitely many existing propositions. ${ }^{38}$ Since these models only have finitely many propositions, they therefore cannot simultaneously satisfy closure of existence under $Q$ and either $\mathrm{K}^{+}$ or $\mathrm{K}^{+}$.

It's natural to investigate models with an infinite variety of existing propositions, and in particular, which satisfy the principle that existence is closed under $Q$. The preceeding model

\footnotetext{
${ }^{35}$ We know that such a bijection exists. Consider, for example, the bijection that maps each finite set $X$ to 2. $\prod_{n} p_{n}^{x_{n}}$ where $p_{n}$ is the $n$th prime and $x_{n+1}$ is the $n$th largest member of $X$ and $x_{0}$ is the size of $X$. And we map $\mathbb{N} \backslash X$ for a finite $X$ to $2 \prod_{n} p_{n}^{x_{n}}+1$.

${ }^{36}$ Given the bijection defined in footnote $35,|Q|(0)=\{\emptyset\}$ and $|Q|(1)=\{W\}$.

${ }^{37}$ We shall show that $\llbracket \phi \rrbracket_{v}$ expresses a finite or cofinite set whenever $v$ assigns only finite or cofinite sets to the propositional variables (thus every closed sentence expresses a finite or cofinite set relative to any assignment). Suppose $\llbracket \phi \rrbracket_{v}$ and $\llbracket \psi \rrbracket_{v}$ are finite or cofinite sets relative to every such assignment $v$. Then any any Boolean combination of them will be finite or cofinite. Given that $R$ is universal, $\llbracket \square \phi \rrbracket_{v}$ will be either $W$ or $\emptyset$ and thus cofinite or finite respectively. Similarly for $\llbracket \phi \equiv \psi \rrbracket_{v}$. If $\llbracket \phi \rrbracket_{v}$ is finite or cofinite for every $v$ assigning finite or cofinite sets, then $\llbracket Q \phi \rrbracket_{v}$ is a singleton, as we noted earlier, which is finite (if $Q^{*}$ is in the language then $\llbracket Q^{*} \phi \rrbracket_{v}$ is the same singleton). Finally if $\llbracket \phi \rrbracket_{v}$ is finite or cofinite for every such $v$ then $\llbracket \forall p \phi \rrbracket_{v}=\llbracket \phi \rrbracket_{u} \cap \llbracket \phi \rrbracket_{u^{\prime}}$ where $u$ and $u^{\prime}$ agree with $v$ except for assigning $W$ and $\emptyset$ to $p$ respectively. Since $u$ and $u^{\prime}$ also only assign finite or cofinite sets to the propositional variables, $\llbracket \phi \rrbracket_{u}, \llbracket \phi \rrbracket_{u^{\prime}}$ and thus $\llbracket \phi \rrbracket_{u} \cap \llbracket \phi \rrbracket_{u^{\prime}}$ are cofinite or finite.

${ }^{38}$ Note that the result that $Q^{n} \top$ expresses a different set of worlds for each $n$ can be derived from $\mathrm{K} 2{ }^{+}$alone, or from $\mathrm{K}^{+}$and the existence of $\top$ and closure of existence under $Q$.
} 
can be adapted to this setting by expanding the domain to include all finite and cofinite sets. As in the earlier models, $Q p$ always expresses a singleton of a world, if $p$ expresses a finite or cofinite set. Thus if $p$ expresses an existing set of worlds, $Q p$ expresses a finite and thus existing set of worlds. Notice also that existence is closed under the finitary Boolean operations, but not the infinitary Boolean operations: a finite combination of unions and complements of some combination of finite and cofinite sets will yield either a finite or a cofinite set, although infinite unions of finite sets might be neither finite nor cofinite.

Notice also that this is a model of $\mathrm{K} 3$ and $\mathrm{K}^{+}$: every finite or cofinite set is the unique member of the extension of $Q$ at some world.

Proposition 4.5. $\mathrm{K}^{+}$has an infinite constant domain regular model in which the propositional domain is closed under finitary Boolean operations and $Q$.

However, the proof that every closed formula expresses a finite or cofinite set breaks down in this setting. ${ }^{39}$ It is no longer clear that this model validates K1 or K2. Indeed, we can show that the Prior sentence expresses an intension that is neither finite nor cofinite for any model of this sort.

Proposition 4.6. Any regular model of $\mathrm{K}^{+}$in which the propositional quantifiers range over the finite and cofinite sets of worlds at each world, and only one intension is in the extension of $Q$ at each world, will be one in which the Prior sentence expresses an infinite and coinfinite set of worlds.

Notice, of course, that by proposition 4.3, any regular model of $\mathrm{K}^{+}{ }^{+}$will be infinite.

For the Prior sentence to correspond to a finite set, the propositions corresponding to each singleton set of worlds must be in nearly every case (i.e. in all but finitely many cases) be $Q$ at the world contained by the singleton. (After all, in these models only one intension is $Q$ at each world, and so if there are infinitely many cases where a singleton is $Q$ at a world that isn't contained by the singleton, then there are infinitely many cases where every proposition that is $Q$ at a world is false.) But that leaves only finitely many worlds to take care of the possibility of uniquely thinking each of the remaining finite and cofinite sets, which is impossible (since there are infinitely many that aren't singletons). What about the hypothesis that the Prior sentence corresponds to a cofinite set? To rule this out, let us consider now not the singleton set of each world but its complement. For the Prior sentence to be true at cofinitely many worlds it must be that in nearly all cases the complement of a singleton be $Q$ at the world contained in the singleton. (After all, if the complement of a singleton is $Q$ at a world contained in that complement then the proposition that is $Q$ is true at the world in which it is thought and so $\lambda$ will be false at such a world.) But if nearly all worlds are such that the complement of their singleton is $Q$ at those worlds, then that leaves only finitely many worlds to take care of the possibility that each remaining finite and cofinite set is $Q$ without anything else being $Q$, which is impossible. ${ }^{40}$ Recall now that it is a feature of our model that the only sets falling in the

\footnotetext{
${ }^{39}$ The crucial step is showing that if $\llbracket \phi \rrbracket_{v}$ expresses a finite or cofinite set relative to any assignment that assigns finite or cofinite sets, $\llbracket \forall p \phi \rrbracket_{v}$ expresses a finite or cofinite set. However if the domain of propositions is infinite, $\llbracket \forall p \phi \rrbracket_{v}$ potentially corresponds to an infinite intersection of sets of the form $\llbracket \phi \rrbracket_{u}$ : even if the latter are all finite or cofinite, an infinite intersection of them needn't be.

${ }^{40}$ More formally, in the model currently under consideration, $\llbracket \lambda \rrbracket=\{w \mid w \notin \sigma(w)\}$. This set is finite if and only if all but finitely many worlds $x \in W$, are members of $\sigma(x)$. Thus all but finitely many singleton sets must be paired with their members according to $\sigma$, which means that there are only finitely many worlds left for the rest of the finite/cofinite sets to be paired with, which is impossible. Similarly, it cannot be cofinite because it would mean that all but finitely sets of the form $W \backslash\{x\}$ get mapped to $x$ under $\sigma$, leaving only finitely many worlds to get mapped to the finite/cofinite sets.
} 
extension of $Q$ are finite or cofinite sets, since $\lambda$ doesn't express either a finite or a cofinite set, the extension of $Q$ won't include the set expressed by $\lambda$. Therefore this model won't satisfy K1, and ipso facto will not satisfy K2. ${ }^{41}$

\subsection{Rich Models and Ramification}

In the above model we've made space for a plenitude of existing contingent propositions, but offered no means for securing K2. Notice also that in the models of K3 given above we have shown that the intension corresponding to any given sentence is the unique member of the extension of $Q$ at some worlds. This doesn't by itself guarantee that there is a possible world at which the proposition that snow is white and the proposition that grass is green are both $Q$ without anything else being $Q$. A further constraint on the models is required if we want to ensure this and similar possibilities. Say that a model is rich if and only if, for any variable assignment, $v$, assigning variables to existing propositions, and set of formulae in the language, $X$, there is some world in the model at which the extension of $Q$ is given by the set of intensions expressed by each member of $X$ relative to $v$.

Here is a recipe for constructing a rich model that also ensures a plenitude of existing contingent propositions, whilst securing $\mathrm{K} 1-\mathrm{K} 3$ and $\mathrm{K} 1^{+}-\mathrm{K}^{+}{ }^{+}$. In these kinds of models we can think of the existing propositions as being arbitrary Boolean combinations of 'basic propositions' concerning only non-intensional matters. Following Kaplan, let's pretend that the basic propositions state facts about the distribution of earth, wind, fire and water (the details don't matter much). We can model these basic propositions as sets of entities we shall call 'proto-worlds'. We shall build up a richer space of possibilities in stages, and hence a space of intensions for formulae, with the proto-worlds corresponding to the possibilities at stage 1. Another layer of possibilities, roughly corresponding to which basic propositions are $Q$ can be generated by considering ordered pairs of proto-worlds with sets of sets of proto-worlds, where the latter component corresponds to the stage 1 intensions that are $Q$ at that possibility. Call these stage 2 possibilities. Now there are more sets of stage 2 possibilities that can be $Q$ than merely sets of proto-worlds, so we must repeat the process. We can generate a third layer - the stage 3 possibilities - by considering ordered triples consisting of a proto-world, a set of sets of stage 1 possibilities (proto-worlds), and a set of sets of stage 2 possibilities. This iterates in the obvious way, with our complete possibilities (or 'worlds') being infinite ( $\omega$-length) sequences where the $n+1$ th component of the sequence is sets of sets of stage $n$ possibilities, giving us the stage $n$ intensions that are $Q$ at that stage.

For a technical reason, that will become apparent later, we also need to stipulate that at each stage, we only add sets of intensions that are 'new' - intensions that couldn't have been generated at an earlier stage. A set of $n$-tuples $X$ is generated by a set of $m$-tuples $Y$ for $m<n$ iff $X$ is the set of all $n$-tuples that extend $m$-tuples in $Y$; thus we will require that the $n+1$ th component of our infinite sequences consist only of sets of $n$-tuples that are not generated by a set of $m$-tuples for smaller $m$.

Our complete intensions are just sets of these infinitary sequences. Notice that our possibilities, as we have constructed them, do not directly tell us which sets of complete intensions are $Q$ at each possibility: they give us an infinite sequence of incomplete (i.e. finite stage)

\footnotetext{
${ }^{41}$ It is relatively easy to tweak the model $M$ so as to get K1 by brute force. Let $\sigma^{*}$ be a bijection between $\mathbb{N} \backslash\{0\}$ and finite and cofinite sets given by $\sigma^{*}(n+1)=\sigma(n)$. For $n>0|Q|(n)=\left\{\sigma^{*}(n)\right\}$, and $|Q|(0)=\{0\} \cup\{n \mid$ $\left.n \notin \sigma^{*}(n)\right\}$. Assuming that the quantifiers range over finite and cofinite sets, we can show that $\lambda$ is vacuously true at 0 , and thus that $\llbracket \lambda \rrbracket=\{0\} \cup\left\{n \mid n \notin \sigma^{*}(n)\right\}$, so this model satisfies $\mathrm{K} 1$ and $\mathrm{K} 1^{+}$. But there is no reason to think that it satisfies $\mathrm{K} 2$ or $\mathrm{K}^{+}$.
} 
intensions, none of which belong to our space of complete intensions. However each stage $n$ intension can be extended to a complete extension in a unique way: if $I$ is a stage $n$ intension, we can consider the set of all infinite sequences in our space, $I^{+}$, that extend the elements of $I$. (Intuitively, we can think of $I^{+}$as an intension that settles some questions about which stage $n$ propositions are $Q$, but leaving it completely open what higher stage intensions are $Q$.) Let us call the extension of a stage $n$ intension to the space of complete intensions a completed stage $n$ intension. Finally, call an intension bounded if and only if it is a completed stage $n$ intension for some finite $n$.

We are now in a position to construct a model. Given any set of proto-worlds, we can construct a set of worlds $W$ consisting of infinitary sequences as described above. As usual we will let our accessibility relation be the universal relation. The domain of the propositional quantifiers, $D(w)$, will be the same at every world: the set of all completions of sets of proto-worlds, i.e., the set of all completed stage 1 intensions. The extension of $Q$ at a world $w$, will contain, for each stage $n$ intension belonging to the $n$th component of $w$, the completion of that stage $n$ intension: $|Q|(w)=\left\{I^{+} \mid \exists n: I \in w(n)\right\}$. So the extension of $Q$ is a set of sets of infinitary sequences, each such set being a completed stage intension that encodes what is $Q$ at a certain particular stage.

Two things are worth noting about this construction. Firstly, the completed stage $n$ intensions are closed under arbitrary Boolean operations. This means in particular that the propositions that exist at each world - completed stage 1 intensions - are closed under arbitrary Boolean operations. Secondly, if $\phi$ expresses a completed stage $n$ intension, and is not a completed stage $m$ intension for any $m<n$, then $Q \phi$ will express a completed stage $n+1$ intension. ${ }^{42}$ Thus in particular the completed stage 1 intensions are not closed under the $Q$ operator.

This model validates $\mathrm{K} 1-\mathrm{K} 3$ and $\mathrm{K} 1^{+}-\mathrm{K} 3^{+}$. We'll show this by demonstrating two things: (i) for every bounded intension there is some world where that intension is uniquely in the extension of $Q$, and (ii) every closed formula expresses a bounded intension. For (i) we can in fact show something stronger: for any set of bounded intensions, there is some world where they are the only intensions in the extension of $Q$. This fact will guarantee that our model is 'rich' in the sense defined earlier. Given a set $X$ of bounded intensions, we can construct a world in the following way: pick any proto world for the first component, and then for the $n$th component of the sequence simply take the set of $n$ stage intensions whose completions are in $X$. The proof of (ii) is a straightforward induction on the complexity of formulae, which we leave to a footnote. ${ }^{43}$

Since the propositional quantifiers range over completed first stage intensions, and thus bounded intensions, (i) guarantees that $\mathrm{K} 3$ and $\mathrm{K}^{+}$are validated. Moreover, given (ii) every closed sentence of the language expresses a bounded intension, so $\mathrm{K} 2$ and $\mathrm{K} 2^{+}$(and thus

\footnotetext{
${ }^{42}$ Suppose that $\llbracket \phi \rrbracket=I^{+}$where $I$ is a set of $n$th stage possibilities (a set of $n$-tuples). Then by construction $\llbracket Q \phi \rrbracket$ is the set of worlds where $I \in w(n)$. (This is where the requirement that $w(n)$ consist only of propositions not generated at earlier stages comes in.) So the set of $n+1$-stage possibilities that contain $I$ in their $n+1$ th component has $\llbracket Q \phi \rrbracket$ as its completion, and so this intension belongs to the $n+1$ th stage.

${ }^{43}$ We shall show that for every formula $\phi$ there is some $n$ such that $\llbracket \phi \|_{v}$ is a completed stage $n$ intension for every choice of $v$ that maps variables to completed stage 1 propositions (and thus a closed sentence will always express a bounded proposition). The inductive hypothesis clearly holds for propositional variables. For each $n$, the completions of stage $n$ intensions are closed under arbitrary Boolean operations. This means that if $\llbracket \phi \rrbracket_{v}$ and $\llbracket \psi \rrbracket_{v}$ are completed stage $n$ propositions for each $v$ mapping variables to completed sets of proto-worlds, $\llbracket \phi \wedge \psi \rrbracket_{v}$ and $\llbracket \neg \phi \rrbracket_{v}$ are also completed stage $n$ intensions. Similarly if $\llbracket \phi \rrbracket_{v}$ is a completed stage $n$ intension for each $v$ mapping variables to completed stage 1 propositions, then $\llbracket \forall p \phi \rrbracket_{v}$ is an intersection of completed stage $n$ intensions, and is thus a completed stage $n$ intension. $\llbracket \square \phi \rrbracket_{v}$ and $\llbracket \phi \equiv \psi \rrbracket_{v}$ are always either $\emptyset$ or $W$, and thus completions of sets of stage 1 intensions. Finally, if $\llbracket \phi \rrbracket_{v}$ is a completed stage $n$ intension for each $v$ of the required type, $\llbracket Q \phi \rrbracket_{v}$ is a completed stage $n+1$ proposition.
} 
$\mathrm{K} 1$ and $\mathrm{K} 1^{+}$) are also validated.

Proposition 4.7. $\mathrm{K}^{+}, \mathrm{K} 2^{+}$, and $\mathrm{K} 3^{+}$have a rich constant domain regular model whose domain contains $T$ and $\perp$ and is closed under arbitrary Boolean operations.

As noted above the domain of the propositional quantifiers is closed under arbitrary Boolean operations at each world, but not under the $Q$ operation. It is possible to tweak the above model so that propositional existence is closed under the $Q$ operation, is closed under finitary Boolean operations but is not in general closed under infinitary Boolean operations. This is achieved by iterating the construction to $\omega_{1}$ instead of $\omega$, and letting the propositional quantifiers range over (completions of) $\omega$-bounded intensions, i.e., intensions that appear at some finite stage. In this model one can show that (i) every $\omega_{1}$-bounded intension (i.e. any intension appearing at a countable stage) is uniquely in the extension of $Q$ at some world and (ii) every closed formula expresses an $\omega_{1}$-bounded intension, guaranteeing the validity of K1-K3 and $\mathrm{K} 1^{+}-\mathrm{K} 3^{+}$. Since the details of this construction are more involved, we omit it.

Proposition 4.8. $\mathrm{K}^{+}, \mathrm{K}^{+}$, and $\mathrm{K}^{+}$have a rich constant domain regular model containing $\top$ and $\perp$ and closed under finitary Boolean operations and closed under $Q$.

The first model we considered above is interesting for another reason: it naturally accommodates the addition of a hierarchy of propositional quantifiers roughly in line with a broadly Russellian approach to higher-order logic. Indeed, for each $n$ we can introduce quantifiers into the language that range over the completed $n$-stage intensions. Such quantifiers will satisfy a restricted version of universal instantiation, guaranteeing that the higher quantifiers range over larger domains of propositions:

$$
\forall_{n} p \phi \rightarrow \phi[\psi / p]
$$

where $Q$ embeds within itself at most $n$ times in $\psi$.

In these models we also obtain a generalisation of $\mathrm{K} 3$ and $\mathrm{K} 3^{+}$for each propositional quantifier, for example:

$$
\forall_{n} p \diamond Q^{*} p
$$

The resulting models bear a family resemblance to Kaplan's description of a construction designed to accommodate $\mathrm{K} 3{ }^{=}$. We'll discuss these connections later in section 7.1.

Augmenting our models in this way thus gives us the following result:

Proposition 4.9. $\mathrm{K}^{+}, \mathrm{K}^{+}$, and $\mathrm{K} 3_{n}^{+}$have a rich constant domain ramified model containing $\top$ and $\perp$ and closed under finitary Boolean operations and closed under $Q$.

\section{Higher-Order Necessitism Revisited}

It's worth drawing attention to the fact that all of the models we have discussed in this section illustrate a theme introduced in section 3, namely that higher-order necessitism (as encoded by NNE, BF and CBF) does not entail UI. In all of our models these three principles are valid, because in each model we chose a constant domain for the propositional quantifiers to range over. However, since in no model was the range of those quantifiers the whole powerset of the domain, we have no reason to think that UI is validated; indeed it can't be validated given that $\mathrm{UI}$ is inconsistent with principles, such as K1, that are validated in these models. 
Notice that in this weaker setting, there is a schematic formulation of necessitism that can't be derived by UI from the principle NNE:

$$
\square \exists p(p \equiv \phi)
$$

Informally, it's necessary that the proposition that $\phi$ exists. This is closely related to a principle of comprehension that is dear to Williamson, and that plays an important role in the book:

$$
\square \exists p \square(p \leftrightarrow \phi)
$$

Indeed, they amount to the same thing when we take $\equiv$ to mean necessary equivalence. This principle is not validated in any of the above models. This is hardly surprising. As noted earlier, UI can be proved from Comp if we assume that identity is necessary equivalence, which is true in all of the above models. Indeed, assuming necessarily equivalent propositions can be distinct Comp may be true even if certain propositions are contingently existing, provided that a proposition necessarily equivalent to it persists through modal space. So, $\mathrm{NNE}^{S}$ may be a better way of regimenting the thought that is motivating Williamson to accept Comp.

In the absence of UI one might therefore wonder whether the combination of NNE, BF and $\mathrm{CBF}$ is the proper way of regimenting higher-order necessitism. However, we think there's no point quibbling about the proper use of the term 'necessitism', especially given that this isn't a term with a long philosophical history. But it is worth noting that there are two separable ideas implicit in Williamson's metaphysical picture. The first is that existence doesn't come and go across metaphysical possibilities; model theoretically, this means that the domain of quantification remains constant between worlds. And this is correctly captured by the combination of NNE, BF, and CBF even in the absence of UI. The second, loosely speaking, is that existence (or its higher-order correlates) is extraordinarily easy, and this is correctly captured by $\mathrm{NNE}^{S}$. If you are thinking that pigs fly, then there's something that you are thinking, and there's some $F$ such that you think that pigs $F$. More generally, the kind of meaning an expression such as, say, a singular term has is such that if it is meaningful, then existential generalisation of the appropriate order into that position is permitted. So, in particular, the kind of meaning a sentence has is such that if a sentence is so meaningful then existential quantification into the position of that sentence is always permitted. This second thought is closely related to the Fregean picture according to which for each grammatical category there is a kind of quantifier that generalises into it, so that a meaningful occupant of that category will be a fit subject for such generalisation. ${ }^{44}$

Regarding the second thought one might think that, even if the quantifiers introduced above are not 'easy' in this sense, it is always possible to formally introduce quantifiers that are, by stipulating that they satisfy universal instantiation. ${ }^{45}$ Similar moves have been made in the context of first-order disputes concerning, for example, the existence of Sherlock Holmes. Were

\footnotetext{
${ }^{44}$ One might think that fictional names are a counterexample to the first-order version of this generalization. Even if Sally thinks that Pegasus flies, there is nothing Sally thinks flies, or so the objection goes. For his part, Williamson suggests that "we should distrust attempts to use fictional or mythological names to refute metaphysical or logical theses. Such terms have a confusing variety of uses [...]. The more such uses are studied, the less they seem to threaten classical theses of logic and metaphysics.' See [34], p. 153. Elsewhere he suggests in a Fregean spirit that he is conducting his inquiry not in ordinary natural language but in a language free of certain defects that afflict natural languages. In this connection a non-referring use of 'Pegasus' is cited as one such defect: 'we should not distort our formal language by allowing for such a term'. See [34], p. 132.

${ }^{45}$ For relevant discussion, see Williamson's [31].
} 
the cogency of such a quantifier automatically conceded, then the main residual dispute would be as to whether the perfectly general quantifier would be interesting, useful, or 'cut at the joints'. But we shouldn't be too quick to assume that such a quantifier is possible to introduce. Granted, for any language, $\mathcal{L}$, it is possible to introduce a quantifier which satisfies universal instantiation of any sentence of $\mathcal{L}$. For example, if we introduce infinitary disjunctions into the language, then one can introduce $\Sigma p \phi$ as a short hand for the infinite disjunction $\bigvee_{\psi \in \mathcal{L}} \phi[\psi / p]$. $\Sigma$ will allow one to existentially generalise sentences of the original language $\mathcal{L}$ provided we have disjunction introduction. However this is not enough to make good on the idea that existence is easy, since we want to be able to generalise into the position that any sentence takes. As defined there will be sentences in the extended language, containing infinitary disjunctions, that we cannot existentially generalise into using the newly defined quantifier (one could repeat this process, but it generate new gaps at each iteration). ${ }^{46}$

Are there general reasons to think that an 'easy' quantifier cannot be stipulatively introduced? We know from the case of 'tonk' that not any old set of stipulations will succeed in introducing a meaningful operator, and in the current context there might be legitimate concerns as to whether these stipulations are satisfiable. While not strictly tonk-like, in that no supplementary principles are needed to derive a contradiction beyond those stipulated to govern tonk, a contradiction will follow from an open ended construal of $\mathrm{K}^{+}$, in which the new quantifiers are allowed to appear as instances.

The models we considered above make good on the first necessitist idea, that existence doesn't 'come and go' across possibilities, but not the second idea according to which existence is easy. One might wonder to what extent this picture is consistent with the spirit of Williamson's view. We won't attempt to judge the issue, but let us mention two considerations that seem pertinent in this regard. Firstly, one of Williamson's arguments for higher-order necessitism stems from the derivability of $\mathrm{NNE}, \mathrm{BF}$, and so on, from the principles such as Comp, $\mathrm{NNE}^{S}$ and universal instantiation. ${ }^{47}$ In other words, Williamson motivates the idea that existence doesn't come and go across possibilities from the thought that existence is easy. Once we've relinquished the latter thought, as we have in these above models, we undercut this way of motivating the former thought, even if we can in fact maintain it in the face of paradox.

The other consideration comes from reflection upon the contingent liar. Consider an ordinary speech involving the word 'says', e.g., 'Everything the Pope says is false' as said by Kaplan at a certain time $t$. There's nothing unusual or self-referential about this speech, and since the Pope does not say anything remotely liar-like, one might think that even if false, in making this speech Kaplan succeeds in saying something. On the other hand, consider a counterfactual scenario in which Kaplan makes this speech at $t$, and in which Kaplan is the Pope. The usual kind of paradox ensues, and the higher-order free logician, for the kinds of reasons we have been examining at length, will maintain that Kaplan doesn't succeed in saying anything. In particular, since Kaplan says that everything the Pope says is false, the proposition that everything the Pope says is false doesn't exist in this counterfactual scenario. Given that for the necessitist, propositions don't 'come and go' into existence, it follows that there is no world at which the proposition that everything the Pope says is false exists. This contradicts the

\footnotetext{
${ }^{46}$ Note that a language which could form disjunctions which contained a disjunct for every instantiation of a sentence of that very same language would not have a well-founded syntax, since the instantiation of the disjunction itself would have to be a disjunct. One could countenance non-well-founded languages like this but they are subject to their own liar-like paradoxes. For example, consider the sentence beginning with an infinite string of negations of ordinal type $\omega$. Such a sentence would be identical to its own negation and paradox would quickly ensue.

${ }^{47}$ See Chapter 6 of [34].
} 
original judgement that the ordinary speech 'everything the Pope says is false' would result in the speaker saying something.

\section{Problems}

\subsection{Paradoxes from Closure Assumptions}

In the models above we noted that the domain of the propositional quantifiers were often closed under some operations but not others. It is natural to wonder whether this is an accident, or if there is an obstacle to satisfying all of the closure principles that we gestured at.

To sharpen matters, let look at what these closure conditions amount to in the object language. Consider the following closure principles:

$$
\begin{gathered}
\exists p(p \equiv \top) \\
\exists p(p \equiv \perp) \\
\forall p \exists q(q \equiv \neg p) \\
\forall p \exists q(q \equiv \square p) \\
\forall p \exists q(q \equiv Q p) \\
\forall p \forall q \exists r(r \equiv(p \wedge q)) \\
\forall p \forall q \exists r(r \equiv(p \equiv q)) \\
\forall p \forall q \exists r(q \equiv \phi \rightarrow(r \equiv \forall p \phi))
\end{gathered}
$$

(where $\phi$ does not contain $q$ or $r$ free).

In all but the last case, the $\mathrm{CL} *$ principle corresponds to closure of the domain under the model theoretic operation corresponding to the connective $*$. In the case of CL $\forall$, closure under arbitrary conjunctions suffices for its truth, although there is no condition statable in the object language under consideration that precisely corresponds to the closure of the domain under arbitrary conjunctions.

Each set of connectives, $X$, the above principles corresponding to $X$ along with the principle $\forall p \phi \rightarrow \exists p \phi$ (a principle ensuring that there's at least one proposition) and the normal axioms governing proposition identity, give rise to a corresponding restricted version of universal instantiation:

$$
\forall p \phi \rightarrow \phi[\psi / p]
$$

where $\psi$ only contains connectives in $X$.

None of our models validated all of these principles. There is a fairly systematic reason for this.

Proposition 6.1. The principles $\mathrm{CL} \top, \mathrm{CL} \neg, \mathrm{CL} Q, \mathrm{CL} \wedge$ and $\mathrm{CL} \forall$ are jointly inconsistent with $\mathrm{K} 1$ and $\mathrm{K} 3$.

Informally, if one has all of the closure principles, one can prove the existence of the Prior proposition from which one can derive a contradiction given K1 or K3 and other background principles. Given $\mathrm{CL} \neg, \mathrm{CL} Q$ and $\mathrm{CL} \wedge$ it is straightforward to show $\forall p \exists r r \equiv(Q p \rightarrow \neg p)$ (noting that $\rightarrow$ is definable from $\wedge$ and $\neg)$. An instance of CL $\forall$ states that $\forall p \forall q \exists r(q \equiv(Q p \rightarrow \neg p) \rightarrow$ $(r \equiv \forall p(Q p \rightarrow \neg p)))$. Then given the existence of at least one proposition (as guaranteed 
by CLT) we can infer from these two things that $\exists r(r \equiv \forall p(Q p \rightarrow \neg p))$. Given that the Prior proposition exists, and the usual axioms governing propositional identity, one can prove a contradiction from K1. Another way to see this is that in the proof of Prior's theorem, we only appealed to an instance of $\mathrm{UI}(\{Q, \neg, \wedge, \forall\})$, and given CLT we can prove $\forall p \phi \rightarrow \exists p \phi$.

Note here that the fact that we are using identity rather than necessary equivalence in the statements of our closure principles plays an important role in this derivation. It could be that for any Boolean combination of existing propositions there is an existing proposition necessarily equivalent to it. In particular, there could be a proposition necessarily equivalent to the Prior proposition. But unless this existing proposition is identical to the Prior proposition, we cannot infer that I'm thinking something from the fact that I'm thinking the Prior proposition.

Closure principles are special instances of general principles telling one how to combine elements from various types to form elements of other types (another example is the principle that for every $x$ and every $F$ there's a $p$ identical to $F a: \forall x \forall F \exists p(p \equiv F a)$.) It's a feature of the free logical approach that it is forced deny a variety of principles of this sort at the type of propositions that are prima facie elegant. Of course K1-K3 are prima facie compelling too, but one wonders whether the gain outweighs the cost.

We should note in passing that there are a number of different kinds of pictures that may be used to flesh out the free logical approach in rather different ways. One kind of constraint is suggested by Russell's vicious circle principle, applied to the propositional type: the constraint that a proposition cannot quantify over a domain that includes itself. But a rather different constraint is suggested by the idea that operators corresponding to intentional attitudes can often take one from the existent to the non-existent. The first constraint is naturally aligned with the rejection of CL $\forall$. Suppose that for every $p$, the proposition that $\square p$ exists, then CL $\forall$ entails that $\exists r(r \equiv \forall p \square p)$ which violates the vicious circle principle: $\forall p \square p$ is in the range of $\exists r$, and thus also in the range of $\forall p$, meaning that $\forall p \square p$ quantifies over a domain that includes itself. ${ }^{48}$ The second constraint naturally leads one to reject CLQ: when $Q$ corresponds to an intentional attitude, the result of combining $Q$ with an existing proposition may take one to the non-existent. Of course within this approach there are all sorts of further decision points. One might think that any sentence containing ' $Q$ ' fails to express an existing proposition, or one might instead think that only the paradoxical ones fail to do so. (If one takes the latter approach one might also very well deny CL $\neg$ since there are sentences for which no paradox attends the assumption that it expresses an existent proposition, but from whose negation a paradox does follow from the correlative existence assumption.) Moreover, the latter approach naturally suggests higher-order contingentism. It will, for example, be a contingent matter whether assuming the existence of the proposition that the guy sitting in room 101 at time $t$ is is thinking only falsehoods leads to paradox. A more necessitist friendly version of the second idea will say that any sentence that could have led to trouble in fact doesn't express an existing proposition.

\subsection{Comprehension and Supervenience}

Whatever one's motivations are for restricting the realm of the existing propositions, it is natural to assume that there remains a realm of fundamental propositions whose existence is unthreatened by intensional paradoxes, or by vicious circle constraints. These may be facts about the field values at particular space-time points, but in the current context no harm will be done to once again assume the imagery of a crude version of pre-Socratic physics, in which

\footnotetext{
${ }^{48}$ This may be a bit quick, but it is beyond the scope of our paper to fully explore the implications of subtly different construals of the vicious circle principle.
} 
the fundamental consists in facts about earth, air, fire and water facts. Further, it appears harmless to assume that arbitrary conjunctions and negations of such propositions similarly exist, for they only concern fundamental matters, and moreover harmless to assume that these propositions exist necessarily if they exist at all.

It is further a metaphysically compelling thought that all truths supervene on these fundamental propositions: that no two possibilities can agree with regard to the truth of all earth, air, fire and water facts but disgree about some intensional matter - e.g. whether I'm thinking something, or about whether everything I'm thinking is false. Once supervenience is assumed, then given our Boolean liberalism regarding to the fundamental propositions it is a short step to saying that every sentence is necessarily equivalent to some fundamental proposition. This in turn entails, given the existence of the fundamental propositions, that every sentence is necessarily equivalent to an existing proposition:

$$
\exists p \square(p \leftrightarrow \phi)
$$

This is, of course, just the comprehension principle Comp we introduced is section 3.

If necessary equivalence guarantees propositional identity, then Comp is tantamount to saying that every sentence expresses an existing proposition, which in turn prevents all the free logical maneuvres for saving K1-K3. But might we move to a hyperintensional setting in which Comp is admitted for supervenience theoretic reasons, but in which K1-K3 are saved by a combination of hyperintensionality and free logic? For example, even given the modal equivalence of some existing proposition, call it 'Fire', with the sentence 'everything I'm thinking is false', our original paradox can be blocked. Given hyperintensionalism and free logic, it's coherent to suppose (i) that I'm thinking that everything I'm thinking is false, (ii) that there's some proposition (i.e. Fire) necessarily equivalent to its being the case that everything I'm thinking is false, and yet (iii) there's no proposition that I'm thinking. Now this is indeed a way of saving K1 while accepting Comp. For example consider a two world model, $W=\{x, y\}, R=\{\langle x, x\rangle,\langle y, y\rangle\},|Q|_{x}=|Q|_{y}=$ $\{W\} \operatorname{Dom}(x)=\{\{\},\{x\}\}, \operatorname{Dom}(y)=\{\{\},\{y\}\}$. Note that since the accessibility relation is the identity relation, $\square$ just amounts to truth, and Comp just amounts to the existence of a true and a false proposition, which is satisfied at both worlds (similarly, propositions exist necessarily for the same reason, even though we have a variable domain model). At both worlds no existing proposition is $Q$ ( $W$ does not exist at either world), even though there are existing intensions necessarily equivalent to the non-existent intension at each world ( $\{x\}$ exists and is necessarily equivalent to $W$ at $x$, similarly for $y$ and $\{y\})$. Since nothing is $Q$ at either world, then $\forall p(Q p \rightarrow \neg p)$ is vacuously true at both worlds, thus has the intension $W$. Since this is $Q$ at both worlds, $\mathrm{K} 1$ (and $\mathrm{K}^{+}$) is true at both worlds. Indeed one can construct (infinite) hyperintensional models that validate $\mathrm{K} 2$ and $\mathrm{K}^{+}$, at least, for the language fragment under consideration. ${ }^{49}$ We can summarize our results with the following proposition:

Proposition 6.2. $\mathrm{K}^{+}$and $\mathrm{K} 2^{+}$are consistent with Comp and higher-order free logic.

But it is instructive to see that even in this hyperintensional setting K3 cannot be salvaged by free logical resources without giving up the assumptions made at the outset. Assume as before that Fire, being a fundamental proposition, exists necessarily, and that it is necessarily

\footnotetext{
${ }^{49}$ The model we describe is not a regular model in the sense of section 4 , since the extension of $Q$ is not given by a set of sets of points, but by a set of closed formulae of the language. Let $W=\mathbb{N}$, and $R=W \times W$, and let $\operatorname{Dom}(n)=\mathcal{P}(\mathbb{N})$. Finally let $\sigma$ be a bijection between $\mathcal{L}$ and $\mathbb{N}$. Truth at a world is defined recursively. For the most part we follow the clauses in section 4. $\llbracket Q \phi \rrbracket_{v}=\{n \mid \phi$ is closed and $n=\sigma(\phi)\}$. We're not offering this as a model that's metaphysically illuminating, but it does establish the consistency of Comp, $\mathrm{K} 2^{+}$and $\mathrm{K} 2$.
} 
equivalent to its being the case that everything I'm thinking is false. K3 requires that it be possible that Fire is thought and that anything else thought has the same truth value as Fire. But whether or not Fire is identical to the proposition that everything that's thought is false, this requirement of K3 is paradoxical. For if Fire is true at the world it is thought, then since Fire is sufficient for its being the case that everything thought is false, then Fire is also false at the world it is thought. And if Fire is false at the world it is thought, and everything thought at that world shares the same truth value as Fire, then everything thought at that world is false. In which case Fire cannot after all be modally equivalent to its being the case that everything thought is false. So in the current setting we have to concede that Fire is a counterexample to K3 and cannot be thought without thinking a proposition with a different truth value. More formally, we've sketched an argument for the following proposition:

Proposition 6.3. K3 is inconsistent with $\square \exists p(\square \exists q(q \equiv p) \wedge \square(p \leftrightarrow \lambda))$ and higher-order free logic.

Note that our assumption strengthens Comp by assuming that the necessarily equivalent proposition to $\lambda$ exists necessarily, which is in line with the thought that fundamental propositions like Fire exist of necessity.

With this concession made the benefits of free logic seem fairly scant. There are a few ways to resist this kind of argument against K3, but all seem a little desperate. One is to deny that arbitrary Boolean combinations of fundamental propositions preserve existence: that is to say, a conjunction, disjunction or negation of earth, fire and water facts might not exist. It could be that every truth is fixed by the distribution of truth values over existing fundamental propositions but if conjunctions and disjunctions of these propositions needn't exist it would be unreasonable to suppose that each truth is modally equivalent to any particular existing proposition. Another move would be to deny that the fundamental propositions exist necessarily. For example, supposing Fire exists and is necessarily equivalent to its being the case that everything thought is false. Perhaps at worlds where I think that everything I think is false and nothing else, Fire doesn't exist.

\subsection{Paradox Without Universal Instantiation}

Free logic provides a way of keeping K1-K3, but K1-K3 are just instances of a wider pattern of intuitive judgments many of which cannot be saved by the free logical maneuvres we've been exploring. For example, while K1 guarantees that it's possible to think $\lambda$ and only thing materially equivalent to $\lambda$, one might think it is similarly possible to think all and only the propositions that are materially equivalent to $\lambda$. We could formalize this idea as follows:

$$
\diamond \forall p(Q p \leftrightarrow(p \leftrightarrow \lambda))
$$

Of course, if many propositions exist, this requires that one think a lot of propositions since $\mathrm{K} 4$ requires that at the witnessing world every proposition or its negation is thought. But it's tempting to think that K4 represents a metaphysical possibility (and this would be particularly plausible if one adopts a lax interpretation of thinking in which it is closed under logical consequences.) However, K4 is inconsistent with the assumption that a truth and a falshood exist - which would be guaranteed if we accepted the minimal principles CL $T$ and CL $\perp .{ }^{50}$ Notice moreover that we can make similar trouble for the weaker hypothesis that one thinks some

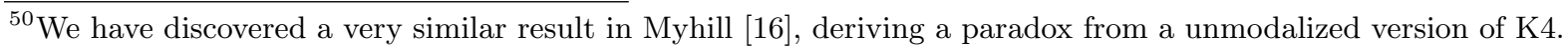


proposition materially equivalent to $\lambda$ and no proposition that is not materially equivalent to $\lambda$ :

$$
\diamond(\exists p(Q p \wedge(p \leftrightarrow \lambda)) \wedge \forall p(Q p \rightarrow(p \leftrightarrow \lambda))
$$

If $\mathrm{K} 5$ is true it is because it is possible to think an (existing) truth and only truths or an (existing) falsehood and only falsehoods. To think an existing truth would mean that it's false that everything thought is false, i.e. $\lambda$ is false, in which case one wouldn't have succeeded in thinking only existing propositions materially equivalent to $\lambda$. Meanwhile, if one thought only existing falsehoods, $\lambda$ would be true, and we would not have succeeded in thinking an existing proposition materially equivalent to $\lambda$ (i.e. an existing truth). This informal argument can be turned into a rigorous proof in propositionally quantified free logic. A closely parallel argument can be run against $\mathrm{K} 4$ and the additional assumption of the existence of a truth and a falsehood (as guaranteed if we accept CL $\top$ and $\mathrm{CL} \perp$ ).

A related observation is that assuming the existence of a truth and a falsehood, there will be interpretations of $Q$ for which a version of Prior's theorem is still provable. Let's interpret $Q$ to mean 'think*', where one thinks* a proposition so long as one thinks a proposition materially equivalent to it. Then one can prove that if one thinks* that everything one thinks* is false, then one thinks* something true and thinks* something false.

It is worth taking a closer look at the Russellian treatment of these paradoxes. Supposing we read the quantifier $\forall p$ as the Russellian's zero level quantifier $\forall_{0} p$, so that the quantifiers appearing in $\lambda$ and the wider scope quantifiers appearing in K4 and K5 are both of zero level. As it stands neither principle violates the vicious circle principle: even though $\lambda$ may express a first level proposition, it's still true that there are zero level propositions materially equivalent to it, and thus that one can divide the zero level propositions into those materially equivalent to it and those not materially equivalent to it. ${ }^{51}$ Given these observations, K4 and K5 - interpreted in terms of zero level quantification - should look just as appealing to the ramifier as anyone else; yet they are plainly inconsistent.

Notice that a proponent of ramification might attempt to retreat to a weaker form of K4 and K5 in which the outer quantifiers range over the same level as the level of the proposition $\lambda$ expresses (which will have to be higher than zero, because $\lambda$ contains zero level propositional quantifiers). Thus, for example, we might weaken K4 to the following principle:

$$
\diamond \forall_{1} p\left(Q p \leftrightarrow\left(p \leftrightarrow \forall_{0} p(Q p \rightarrow \neg p)\right)\right)
$$

Unfortunately this principle is also problematic. Assume, as customary, that every zero level proposition is a first level proposition (that is, the levels are 'cumulative'), and assume also that there is at least one zero level truth, and at least one zero level falsehood. Consider the world that witnesses the truth of $\mathrm{K}^{-}$. Either I'm thinking a zero level truth or I'm not at this world. If I am, then $\forall_{0} p(Q p \rightarrow \neg p)$ is not the case, thus if I'm only thinking first level propositions materially equivalent to $\forall_{0} p(Q p \rightarrow \neg p)$ I'm only thinking first level falsehoods. But by assumption I'm thinking a zero level truth, and thus a first level truth since every zero level proposition is a first level proposition. On the other hand, suppose that I'm not thinking any zero level truths. This gives us the truth of $\forall_{0} p(Q p \rightarrow \neg p)$, which means, since I'm thinking all and only the first level propositions materially equivalent to $\forall_{0} p(Q p \rightarrow \neg p)$, that I'm thinking every first level truth, including the zero level truth, contradicting our assumption.

\footnotetext{
${ }^{51}$ One could assert that no zero level propositions are materially equivalent to $\lambda$ by denying the existence of any zero level propositions. Or one could go for a very radical form of ramification in which the truth functional connectives themselves have a hierarchical structure.
} 
Given all of these observations one might wonder just how much we have really achieved by moving to higher-order free logic. The proponent must find a principled background picture that motivates K1 (say) without also motivating the outright inconsistent principles $\mathrm{K} 4$ and K5. Here is one way you might attempt to flesh out that picture: one could argue that thinking $\lambda$ (and nothing else) is an easy matter. All one needs to do to achieve that is to mentally token something corresponding to the sentence 'everything I'm thinking is false', without tokening anything else at the same time. By contrast, it is not so easy to ensure the existence of a proposition by performing these mental tokenings - the existence of propositions is beyond your mental powers. This picture naturally motivates K1 (and indeed K2 if we generalise to other sentences), but note that it does not motivate $\mathrm{K} 5$ since $\mathrm{K} 5$ requires it to be possible for you to think something existing materially equivalent to $\lambda$ - tokening $\lambda$ does not guarantee the existence of anything. Similarly, it's unclear what you could mentally token in order to ensure the truth of K4: since it is beyond your control which propositions exist merely tokening a number of sentences does nothing to guarantee that you think all and only the existing propositions materially equivalent to $\lambda$.

Although one can in principle outline a picture in which K1 and K2 are motivated without also motivating $\mathrm{K} 4$ and $\mathrm{K} 5$, we feel that once one has stopped taking these kinds of compelling judgments at face value, one wonders whether we shouldn't just accept the original consequences of UI for K1 and K2.

\subsection{Expressive Limitations}

Given UI one can in a higher-order framework make extremely general claims by recourse to higher-order quantification. For example, if we think that knowledge is factive one can capture this idea quite straightforwardly with the following sentence:

$$
\forall p(K p \rightarrow p)
$$

Similarly one can state that all conjunctions have true conjuncts in a way that is not limited to the conjunctions expressable in any particular language:

$$
\forall p \forall q(p \wedge q \rightarrow p)
$$

Now even when we relinquish UI we may write down these things, and remain confident in them. But in a free logical setting they may feel insufficiently general. For example, supposing there is no such proposition as $\lambda$, we may feel the urge to write down generalisations for which the following are special cases:

$$
\begin{aligned}
& K \lambda \rightarrow \lambda \\
& \lambda \wedge \forall p p \rightarrow \lambda
\end{aligned}
$$

However the quantified sentences specified above are unfit for this purpose, because they do not entail these instances.

A natural thing to try to go for is a schema rather than a quantified claim. For example the factivity of knowledge could be captured by the following schema:

$$
K A \rightarrow A
$$

An initial concern with this strategy is that it won't satisfy our desire to write down a general truth, since schemas are neither true nor false (even though they can have true or false 
instances). ${ }^{52}$ Note that this cannot be readily circumvented by instead writing down a metalinguistic generalisation to the effect that all uniform interpretations of the schematic letters deliver truths, since this reintroduces propositional quantification and hence may not have the requisite generality.

In order to avoid quantification over interpretations of sentence letters one might opt for a metalinguistic generalisation that is substitutional in nature, along the following lines: every uniform substitution of a schematic sentence letter by a meaningful sentence in the language yields a truth. But this would lose generality for a different reason, since, for example, the generalisation about factivity is supposed to cover cases of knowledge that aren't expressible in our language. A similar concern would attach to any effort to express the required generalisations using infinitary conjunctions or disjunctions of sentences in our finitary language. Note that arguably no infinite conjunction can contain all sentences of the infinitary language as conjuncts, for then it would contain itself as a conjunct and thus be non-wellfounded.

Thirdly, quite aside from the desire to express bona fide truths, the schematic approach is particularly problematic in certain embedded environments. Take the generalisation that in the classical setting we would happily express with the following formula:

$$
\diamond \forall p Q p
$$

I.e., it is possible that every proposition is $Q$. Now suppose we felt this inadequate because we wished to convey the thought that it's possible to think, alongside all the existing propositions, $\lambda$, and $\neg \lambda$ and so on, whether or not the latter correspond to existing propositions. In this connection the following schema will not be of any use:

$$
\diamond Q A
$$

After all, that schema does not require that $\lambda$ and $\neg \lambda$ be thought together in any single world. This point generalises to occurrences of universal quantifiers in embedded environments where, in the classical setting, the embedded universal quantifier wouldn't commute with the embedding.

One might hope to bypass a number of these concerns by appeal to a device alluded to earlier. One might try to express some of these generalisations using ellipsis in the following kind of way:

If you know that snow is white then snow is white and if you know that grass is green then grass is green and ...

Note that for this to do the requisite work the '...' cannot merely be a stand in for an infinitary conjunction of sentences in the finitary language for this lacks the generality we were seeking. The ellipsis must, for example, be understood at least to cover all possible extensions of the language. So let us assume that the object language has been extended to include exactly this kind of ellipsis.

There are a number of pressing concerns here. One is that the more careful one is about the syntax of this device, the more it begins to resemble a quantifier. For example, one will have to introduce a variety of '...'s corresponding to the need for different propositional variables in the original setting. But we'll focus on a particular dilemma. Are '...' thoughts to be understood so as to be under the scope of '...' generalisations? For example, is the claim that if I know

\footnotetext{
${ }^{52}$ Note that sometimes philosophers adopt the convention of calling a schema true if its universal closure is true (where each schematic letter is bound by a quantifier of the requisite type), but that won't help in the relevant setting since it will send us headlong into the first problem of insufficient generality.
} 
that grass in green then grass is green and ... to include as a special case that if I know that [if I know that grass is green then grass is green and ...] then [if I know that grass is green then grass is green and ...]? If the ellipsis is indeed a legitimate extension of the language, and '...' claims are supposed to cover all legitimate extensions of the language then '...' claims had better be special instances of '...' claims. An initial worry is that this raises the spectre of non-wellfoundedness. But more importantly, the '...' claim seems now to behave enough like a classical quantifier as to allow one to reinstate the Priorian paradoxes. Consider K1․

$\mathrm{K} 1 \cdots \diamond(Q((Q \top \rightarrow \neg \top) \wedge \ldots) \wedge(Q \top \rightarrow(\top \leftrightarrow(Q \top \rightarrow \neg \top) \wedge \ldots)) \wedge \ldots)$

Once one has gotten the hang of ellipsis, K1* should look just as compelling as the original K1. Note that from $\phi(T) \wedge \phi(\perp) \wedge \ldots$ one can conclude $\phi(\psi)$ for any $\psi$ in our language (whether $\psi$ includes ellipsis or not). This principle is exactly analogous to the principle of universal instantiation that caused problems for K1 in the first place.

\subsection{First-Order Free Logic}

We've have in effect been developing a pessimistic pictures of the prospects for a higher-order free logic that's motivated by the prospect of keeping principles like K1-K3. It less clear whether the materials of this section can be developed into a case against higher-order free logical approaches that are motivated by other concerns (for example, by the desire to be a contingentist about the existence of properties). Moreover, it's even less clear whether these considerations carry over to the first-order setting. For example, concerns about closure under Boolean operations have no analogue in the domain of individuals, nor do concerns about supervenience. Of course expressivity worries, and in particular the challenge of finding suitable ways of formulating perfectly general principles, are likely to be a common theme among those who oppose free logical approaches in general.

We are not going to systematically explore the resources of various free logicians for enhancing expressive power. But we will mention one theme that makes things particularly difficult for one who promotes free logic as a means for saving K1-K3. Suppose, for example, one is a first-order free logician. One might still be attracted to the schema $\diamond \exists x(x \equiv t)$, for that allows one to use a modalised quantifier to formulate perfectly general claims, for which singular terms are instances. Similarly, one might pursue such a strategy for a higher-order free logic. However, such a strategy is dangerous, to say the least, if ones motivation for higher-order free logic is the preservation of K1-K3: $\diamond \exists p(p \equiv \phi)$. This is because modalised quantifiers, once accepted, may well come to form the basis for revenge-like versions of Prior's paradox.

\section{Kripke, Kaplan and Ramification}

Let us now compare the free-logical approach to the Prior-Kaplan paradox to another prominent family of approaches, based on the Russellian, as opposed to the Fregean, tradition in higher-order logic. As noted at the outset both approaches raise complications for the principle of universal instantiation. The Russellian tradition deploys the machinery of ramification. In Russell's work this ramification is relatively thorough going: every relational type over arguments of type $\tau_{1}, . ., \tau_{n}$, is stratified into an infinite hierarchy of levels indexed by natural numbers, with exceptions for certain logical vocabulary. ${ }^{53}$ Complex grammatical expressions

\footnotetext{
${ }^{53}$ Note that when this is spelled out fully, an unary operator (for example) is indexed by a pair of numbers: one that signifies the level of the input proposition and the other that signifies the level of the output proposition. Recalling the notation from the introduction, we would represent this by the ramified type $(() / n) / m$.
} 
are built up in such a way that no non-logical grammatical expression applies to an expression of an equal or higher level - in other words, the hierarchy of levels is cumulative: so for example an operator with ramified type $(() / 4) / 5$ can apply to propositions of types ()$/ i$ for $i \leq 4$. It should be noted that Kaplan himself is working squarely within this tradition.

Of course one might explore more relaxed ramified approaches. We have already noted that some expressions, namely the logical expressions, are not subject to stratification. In what follows we'll explore a range of less stringent stratificational approaches to the Prior-Kaplan paradoxes, by way of attempting to isolate the benefits of particular stratificational maneuvres.

There are some important choice-points facing the ramifier. One concerns the effects of level mismatch. Suppose, for example, a predicate taking arguments of level two - e.g. a predicate of ramified type $(e / 2) / 3$ - is combined with a name of level five - i.e. $e / 5$. Is the resulting string simply ungrammatical, with no truth value, or is it a grammatical sentences that is simply false (and whose negation is true)? Russell (and Kaplan) take the former route, but one might explore the falsity route as well. For example, many proponents of the idea of indefinite extensibility argue that there is a hierarchy of domains for the first-order quantifiers, which we might label $e / 0, e / 1$ and so on. If this kind of theorist is not also stratifying propositions - something we will consider shortly - then relational types need not be stratified (so that if $\tau_{1}, \ldots, \tau_{n}$ are types $\left(\tau_{1}, \ldots, \tau_{n}\right)$ is a type). In one context we can predicate a property $F$, of type $(e / 0)$, of every member of the initial domain, $e / 0$. But from another context one can quantify more expansively - over $e / 1$ individuals, say - and assert that some $e / 1$ individuals don't have $F$. This would require applying $F$, of type $(e / 0)$ to a variable ranging over $e / 1$ entities and thus the grammaticality (albeit falsity) of a level mismatch. There are also proponents of indefinite extensibility concerning quantification into sentence position, introduced so as to deal with the liar paradox (see Parsons [17], and Glanzberg [8]). If you are stratifying both individual and propositional types then both the individual and relational types must have levels, and we are in a version of the Russellian framework modulo this interpretation of level mismatch. ${ }^{54}$

It's perhaps worth noting that the ungrammaticalist might try to retain a facsimile of universal instantiation, for they claim that all of its grammatical instances are valid, although this defensive strategy is less promising than it might initially seem (we'll return to this point later). ${ }^{55}$

A second choice point concerns the extent to which we ramify. As noted by Russell, and Kaplan after him, we could ramify all relational types (with the exception of a small list of logical operators). Particularly relevant in the language fragment we outlined in section 2 is that both the operators $Q$ and $\square$ will be ramified into an infinite family of operators with different ramified types. The quantifier phrase $\forall p$ will similarly come in a family of different levels depending on the ramified type of the variable $p .{ }^{56}$ But less thorough going ramification

\footnotetext{
${ }^{54}$ We note in passing that some implementations of Tarski's stratificational approach to the liar adopts this second tack: for example he endorses the schema $\neg \operatorname{Tr}_{n}(\ulcorner\phi\urcorner)$ when $\phi$ contains a truth predicate of level $n$ or higher.

${ }^{55}$ The most straightforward definition of sharing a grammatical category has to do with substitution preserving grammaticality. Unfortunately this is not available to someone who takes type mismatch to induce ungrammaticality since, for example, switching names of different types may turn a grammatical sentence into an ungrammatical one, even though all names belong to the same grammatical category. Here is an alternative definition that is available to such a theorist, at least if they opt for a cumulative approach: two expressions belong to the same grammatical category just in case there is some expression such that both are in the range of its significance. If instead one goes non-cumulative, it's no longer clear how to make good on the vision that one is stratifying within a grammatical category, since the notion of grammatical category is no longer clear. Such a view arguably collapses into Fregeanism.

${ }^{56}$ As noted in the introduction, on standard ways of setting up the framework, the expressions of the form $\forall p$ can be ordered by the natural numbers because the variable $p$ can be assigned a ramified level - the expression $\forall$
} 
could also be entertained. One might ramify quantifiers for reasons having to do with the vicious circle principle, without thereby feeling any particular pressure to ramify attitudes or modal operators. The vicious circle principle, as Russell articulates it, requires that 'whatever contains an apparent variable must not be a possible value of that variable. Thus whatever contains an apparent variable must be of a different type from the possible values of that variable; we will say that it is of a higher type.' Whatever Russell's own view, one might think that attitudinal operators do not contain 'apparent variables', and therefore will not require ramification. ${ }^{57}$

To pursue this sort of approach, we would simply treat $Q$ and $\square$ as syncategorematic, in the same way that we treat $\neg$. One important upshot of this relaxed stratificational approach is that we can iterate these operators so that, for example, the formula $\square \square(p \rightarrow p)$ is well-formed (and, indeed, true). Since there are now no expressions in our language for which level mismatch can arise, we effectively have a classical language that just contains an infinite number of distinct quantifier phrases $\forall p_{0}, \forall p_{1}, \forall p_{2}, \ldots$

To illuminate each approach we'll also see how each applies to some puzzles related to one discussed in Kripke [12] (and for which Kripke also entertains a stratificational solution). Kripke imagines that, at a certain time $t$, someone undertakes to think uniquely about the set of times, $t^{\prime}$, at which there is some set of times being thought about at $t^{\prime}$ that doesn't contain $t^{\prime}$. On the one hand it seems as though that person is uniquely thinking about this set at $t$. But on the other there seems to be a logical difficulty attaching to such a supposition, for there is now no coherent answer to the question whether the set thought about contains $t$.

To begin it's worth noting that there are some uninteresting puzzles in the vicinity for which an appeal to ramification seems excessive. Suppose at noon on each day of sixteen days I stand on a different square of a large square chessboard. On each day I'm given the opportunity to touch some or all of the squares on a chess board. Now there seems to be a logical guarantee that on none of the sixteen days will I touch all and only those squares which have the property of being not among the squares I touch when I'm standing on it. Nothing is particularly puzzling here - it turns on such mundane facts such as the fact that I can't touch on Tuesday the squares I am not touching on Tuesday - and certainly nothing that calls for anything as high powered as ramification.

There are some observations about thinking that are equally mundane. Take, for example, the wide scope construal of the sentence 'he's thinking about the smallest number that he's never thought about' viz 'there's a unique number, $n$, such that $n$ is the smallest number that he's never thought about, and he's thinking about $n$ '. It is unsurprising that there's a logical guarantee that he is not thinking this number, and it has nothing to do with thinking - try kicking the smallest policeman you'll never kick!

But let's turn to a more puzzling version of the chessboard puzzle. Let's introduce a relation, touching*, where you touch* a square by touching a predicate that expresses a property possessed by the square. In the new protocol you have a book of predicates, and you touch one or more predicates from the book whenever you stand on a square. Now suppose on a given day you touch just one predicate which expresses the property of being a square that you don't touch* on that day (suppose it's Tuesday and the book contains the predicate 'being a square that you don't touch* on Tuesday'). For each square we can derive a contradiction from the hy-

itself is syncategorematic.

${ }^{57}$ One typically pursues this idea by ramifying the variables instead of the quantifiers, by insisting that a variable of type $\tau$ be only assigned values in a particular domain, and treating quantifiers themselves as type neutral rather like Russellian negation (as noted above). One could also achieve a similar effect by having a single type of variable, but by ramifying the device we use to $\lambda$ abstract. It is unclear to us what, if anything, is at stake between these variants. 
pothesis that you don't touch* it, for then you would satisfy a sufficient condition for touching* it (namely touching a predicate that expresses a property instantiated by the square). And we can also derive a contradiction from the assumption that you do touch* it, and the assumption that you've touched a predicate that doesn't express any other properties, for then you would fail a necessary condition for touching* it. It would be very unsatisfying to conclude from these logical reflections that in scenarios such as this there would be a force-field surrounding the relevant predicate token in the book, so that if you were to try to touch it you'd be repelled, or suffer other similar physical mishap. Similarly, it would be unsatisfying to conclude, with respect to the puzzles about thinking, that there are force-fields that block the appearance of certain mentalese sentences in one's belief box, or in some other cognitive module.

Let's contrast two very different approaches roughly corresponding to differing ways of ramifying gestured at above. On one approach vicious circle considerations prohibit such properties as the property of being a square that does not instantiate any property expressed by a touched predicate, with the quantifier naïvely construed as ranging unrestrictedly over all properties. For such a property would then quantify over a domain that includes itself. One might thus only tolerate properties that quantify over a limited domain of properties that do not contain themselves. For example, there will be properties like being a square that does not instantiate any colour property expressed by a touched predicate, where that property is not itself a colour property. Supposing one touches a predicate expressing that property and nothing else, there is no puzzle about what to say: in touching that predicate, one thereby touches* all the squares. ${ }^{58}$ Note that there is no need here to ramify the relation of expressing; all the work is done by applying vicious circle considerations to quantification over properties.

There is a very different approach that may be considered. To make it vivid, let's unpack the predicate 'being a square that isn't touched* on Tuesday' as follows: 'being a square that doesn't instantiate a property expressed by a predicate that is touched on Tuesday'. Supposing one touches that predicate on Tuesday. For a paradox to arise we need, among other things, to suppose that 'express' expresses the expressing relation. So one strategy for blocking the puzzle is to deny that in that context the predicate 'expressing' as it appears in the book expresses the expressing relation. Of course, we can allow that it expresses something - perhaps there is another relation, expressing* that 'express' in that context expresses. Suppose that in the book the predicate 'being a square not possessing any property expressed by a property that Lucifer touches' occurs in the book. Supposing Lucifer touches nothing, there is no immediate paradox in supposing that 'expresses' as it occurs in that predicate expresses the expressing relation, but we may be attracted to a uniformity assumption according to which at a context 'expresses' is uniform with regard to which relation it expresses, and is not at the semantic mercy within a context of its linguistic surroundings. This kind of consideration may well encourage one to think that no token of 'that token of 'expresses' expresses expressing' is true. ${ }^{59}$

Of course, these anti-disquotational thoughts are one thing, but the claim there is a family of expressing-like relations that form a hierarchy, of the kind envisaged by typical ramifiers, is a further commitment. Justifying this will require inter alia blocking loops (so that, for example, we don't allow that 'expresses' expresses expressing* and 'expresses' expresses* expressing), and infinite descending sequences, thereby securing well-foundedness. ${ }^{60}$ Notice that even if a

\footnotetext{
${ }^{58}$ This seems very much in line with a solution entertained, albeit in a compressed way, by Kripke to his own puzzle.

${ }^{59}$ As argued in a lot more detail in Bacon [1].

${ }^{60}$ The ordering technique we have in mind is one according to which if 'expresses' expresses' expressing', then expressing' ' comes immediately below expressing' in the ordering. Ruling out loops and infinite descending sequences together would still guarantee no more than a partial order; further arguments would be needed to
} 
well-ordered hierarchy of expressing-like relations were justified that would still not yet secure some of the standard prohibitions voiced by the ramifiers. For example, a typical ramifier for 'expressing' will contend that whenever two tokens of 'expresses' express the same relation and one occurs within the scope of another then the result is either false or meaningless. Even given the hierarchy, and even given that no token of 'expressing' ever stands in some relation from the hierarchy to itself, this further commitment is far from secure. Suppose for example that one had a coarse grained conception of propositions. Then, since ' $2+2=4$ ' expresses the proposition that $2+2=4$, and since the proposition that $2+2=4$ just is the proposition that ' $\mathrm{dog}$ ' expresses something or it doesn't (i.e. the one and only necessary proposition) then ' $2+2=4$ ' expresses the proposition that 'dog' expresses something or it doesn't. This would then give us a counterexample to the aforementioned ramificationist dictum, one that doesn't require us thinking that 'expresses' ever expresses expressing. In particular, it may be that there is some relation other than expressing, namely expressing*, that is expressed by each occurrence of 'expresses' in the previous example. For these reasons the postulated hierarchy does not yield any straightforward syntactic constraints connecting embeddings or relative scope to the possibility of truth or meaningfulness (indeed, if one takes a coarse-grained approach, there could not be any such constraints). Note that this hierarchical vision does not involve any requirement that property quantification be somehow restricted or stratified.

Kaplan has suggested that once one has embraced ramification, one should be unhappy with the fundamental structure of possible world semantics. While this is not the place to pursue the matter at length, it's worth noting that this sort of stratification just described does not indicate a fundamental difficulty for possible world semantics. As we see things, Kaplan's concern with naïve possible world semantics is that it assumes the domain of propositions can be given 'all at once' - after all, possible world semantics takes propositions to be identical to or determined by sets taken from an antecedently given domain of worlds. ${ }^{61}$ And it is certainly true that once one ramifies propositional quantification this aspect of possible world semantics is called into question. However, if one leaves quantification alone but instead appeals to a hierarchy of expressing relations, or thinking relations, then no difficulty will arise from the idea that one can propositionally quantify over all propositions at once. So from this second perspective possible worlds semantics may survive completely unscathed. ${ }^{62}$

\subsection{Ramification and Prior-Kaplan Puzzles}

We've distinguished some varieties of ramification, but how do these varieties interact with K1-K3? Let's examine in turn first the strategy of ramifying the attitudinal operators and then the strategy of quantificational ramification. We'll then examine whether there are special advantages to ramifying along both fronts when it comes to the Prior-Kaplan puzzles.

Suppose first one leaves higher-order quantification unramified, but does ramify operators like 'it is thought that'. ${ }^{63}$ We'll focus here on the standard kind of ramifier, that prohibits the

secure the kind of well-order that would justify indexing by the natural numbers.

${ }^{61}$ Even if one took Kaplan's lessons to heart this wouldn't make trouble for the models that we employed in section 4. Note, importantly, that Kaplan makes it definitive of possible world semantics that one treats the object level propositional quantifiers as ranging over all sets of worlds, but note that the models of section 4 therefore do not count as 'possible worlds' models in that sense. There is more to be said about the relation between possible world semantics, as Kaplan describes, and what typically goes under the name 'possible worlds model theory', but we are not going to pursue these issues further here.

${ }^{62}$ Of course for reasons given earlier, hardcore syntactic constraints on truthfulness and meaningfulness adopted by some ramifiers do not sit well with coarse-grained theories of propositions that are often adopted within possible worlds semantics.

${ }^{63}$ This could be achieved by stipulating that all propositional variables have the type ()/0: this effectively means 
truth of any sentence that has an attitudinal operator appear within either its own scope, or the scope of an operator of lower type (as noted, there is a further divide among those who think that such occurrences are either false or ungrammatical). ${ }^{64}$ Obviously this framework won't validate $\mathrm{K} 1$ on any uniform interpretation of ' $Q$ ', for $Q$ appears within its own scope in K1 (and so it is either false or ungrammatical). Generally, when one writes down a schematic principle one intends the schematic variables to be interpreted uniformly. As applied to K1, it seems as though we shouldn't have been writing it down after all. That said, the present kind of ramifier can offer a diagnosis of why claims like K1 seem attractive. It's natural for the proponent of this view to contend that when, for example, in ordinary thought and talk we say things like 'I thought Bob thought it was Tuesday' we don't automatically say something false or incoherent. Rather, without properly keeping track of what we're doing: the two occurrences of 'thought' correspond to two things at the level of logical form. From this perspective there's a kind of logical form 'blindness' that ordinary people are susceptible to, and which could explain why they would mistake K1 for a family of well-typed principles that could be true. Indeed, it's easy to find models in which one can uniquely think $k_{2}$ the proposition that everything I think $k_{1}$ is false. ${ }^{65}$

This style of explanation can't be easily extended to the puzzles associated with K3, or the variant Kaplan discusses, $\mathrm{K} 3^{=}: \forall p \diamond(Q p \wedge \forall q(Q q \rightarrow q=p))$. Both principles are well-typed by the lights of the ramifier. Moreover, Kaplan's original considerations telling against $\mathrm{K} 3{ }^{=}$seem to be just as compelling in this setting. There are still many more propositions than worlds, so not every proposition can be uniquely thought ${ }_{1}$ - the fact that there will be a whole hierarchy of additional unsatisfiable principles is of little comfort. As for K3, one cannot run Prior's original argument, because certain steps in the proof violate the typing constraints. However, Prior's original argument can be adapted so as to respect those typing constraints, e.g. by saying things like 'there's something identical to the proposition that everything I'm thinking ${ }_{1}$ is false, and I could have thought ${ }_{1}$ it and only propositions materially equivalent to it'. One could block this trick by taking seriously the idea that the quantifiers are typed, and thus that the variable flanking the right hand side of the identity connective cannot appear under the scope of 'thinks 1 ' since it must be of the same level as the sentence on the left hand side ('everything I'm thinking 1 is false' which is of level 2). But this would no longer be the selective kind of ramification under consideration. ${ }^{66}$

What about K2? A natural thing for the ramifier to try is to say that K2 is true for any well-typed instance. ${ }^{67}$ A modification of the model construction in proposition 4.7 from section

quantification over propositions doesn't come in levels, but the operators still get ramified types of the form $(() / n) / m$ for $n<m$ and so cannot be iterated. If we are going for a falsity interpretation of level mismatch, we would not require any special syntactic restrictions.

${ }^{64} \mathrm{Much}$ of what we have to say can be adapted to the view that there is a hierarchy of thinking relations, but no straightforward syntactic scope constraint concerning when attitudinal verbs can be embedded within one another to produce truths.

${ }^{65}$ For example let $W=\{w\}, D(w)=\{\emptyset,\{w\}\},\left|Q_{1}\right|_{w}=\emptyset,\left|Q_{2}\right|_{w}=\{\{w\}\}$.

${ }^{66}$ For what it's worth we do recognize that there may be something a little unstable about a ramification strategy that imposes austere syntactic constraints on attitudinal verbs, but does not ramify the variables or quantifiers. One effect of this combination is that one can have syntactically well formed instances of the premises of Leibniz's law, such as $Q p$ and $p=Q q$ but ungrammatical instances of the conclusion, such as $Q Q q$. But as we see it there is no similar instability attaching to a view that espouses a hierarchy of relations but imposes no syntactical prohibitions. In other respects the view might make similar moves to the more traditional ramifier, for example, with the traditional ramifier it may think that our inclination to the hypothesis 'it's possible to uniquely think that everything you're thinking is false' or 'it's possible for it to uniquely seem as though you're saying that everything it seems as though you're saying is false' is based on a blindness to the underlying structure of our mental states and speech acts.

${ }^{67} \mathrm{On}$ the interpretation on which type mismatch induces ungrammaticality, this interpretation of K2 is especially 
4.3 serves to show this is consistent. ${ }^{68}$ On this way of understanding K2, K1 isn't an instance of it, since it violates the typing constraints. In this setting the supervenience problems discussed in section 6.2 are still relevant: supposing that thinking facts supervene on earth, fire, wind and water facts, then for all we know there's an earth, fire, wind and water sentence that expresses a proposition that's necessary and sufficient for thinking that everything I'm thinking is false (of course, supervenience doesn't guarantee that there is such a sentence). The instance of K2 with this sentence would not violate typing constraints, but as we saw in section 6.2 , this instance would be false. That said, one might, taking a leaf from Kaplan book, have the more modest ambition of saving the logical coherence of K2, rather than the truth, and in particular, its compatibility with various plausible metaphysical hypotheses. ${ }^{69}$

Let us turn to the ramifier who selectively ramifies quantification, but does not ramify the attitudinal operators. There are issues of detail concerning whether it is the quantifiers, variables, or lambda operator that are to be ramified here; we won't fuss about this, but for simplicity we will work with a picture in which it is the variables that get ramified and that determiners like 'all' are no more typed than logical operations such as 'not'. (Moreover, while the abstract idea of typing quantifiers does not force any kind of cumulative hierarchy, we'll imagine, with the standard ramifier, that the domains of these quantifiers are linearly ordered by inclusion.) ${ }^{70}$

By contrast with the last strategy discussed, this kind of ramifier would with Kaplan have legitimate concerns about possible world semantics as it is standardly understood. Possible world semantics encourages one to think that from within one's language can quantify 'all at once' over a complete and unextendable domain of propositions. But for this kind of ramifier, this is an illusion: each quantifier ranges over a domain of propositions, each of which may have maximally strong elements. For each level of quantifier, these maximally strong elements become stronger and stronger with no end - while possible worlds may be the limit of these elements, there is no way of simulating quantification over these limits. ${ }^{71}$

What happens to K1-K3 on this interpretation? As noted in section ??, if the different levels of quantification are not to collapse into one another, then each quantifier must abide by a free logic (this is due to Harris's theorem). ${ }^{72}$ What bears emphasis is the tight connection between the ramified quantifier picture and the discussion of free logic in the earlier sections of this paper. For note that on any interpretation of the quantifiers universal instantiation - and coordinately existential generalisation - will fail in just the kind of way discussed in those sections. Thus, if I'm saying that everything ${ }_{1}$ I'm saying is false, there's nothing ${ }_{1}$ (although something 2 ) that

natural as we don't in general consider ungrammatical substitution instances of schematic principles.

${ }^{68}$ We let the quantifiers range over bounded propositions, and the extension of $Q_{n}$ is the extension of $Q$ (as defined in section 4.3) intersected with the $n$ level propositions. To see that this model works one first shows that if the highest type in $\phi$ is $n$ then $\phi$ expresses an $n$ level intension. Thus every sentence whose type does not exceed $n$ expresses an $n$ level intension, and so is $Q_{n+1}$ at some world.

${ }^{69}$ For our part, we don't see any special appeal to a view that says K2 is obviously logically coherent, but not the conjunction of K2 with a supervenience thesis. Why not say instead that the logical mastermind would pour scorn on both on account of Prior's theorem?

${ }^{70}$ We might note in passing that, although not in the Russellian tradition, one might want to sort first-order variables into types with disjoint domains (see McDaniel [14]). One might also envisage cross cutting domains - a picture encouraged by some of the literature on quantifier variance. Talk of 'domains of quantification' here and in the text is of course a bit of cheat (see footnote 7.1 below)

${ }^{71}$ Even this characterisation of how the quantifiers relate to each other is something of a cheat, since it pretends to be able to talk all at once about all the levels, yet we have no quantifier that can do this. This kind of consideration motivates Williamson [33] to think that absolutely general first-order quantification is unavoidable; arguably these considerations generalise to quantification into sentence position.

${ }^{72}$ In this framework - where operators and connectives aren't typed - it's not so obvious whether any type mismatches can occur, since a variable cannot be in the scope of another. Thus the exact treatment of type mismatches doesn't matter so much in this context. 
I'm saying; and similar kinds of results can be produced for the quantifier 'something,', and so on. So each quantifier is governed by a positive free logic. Note that in those sections, as here, we are not typing 'thinks' in such a way that prohibits it appearing within its own scope.

It is worth noting that in section 4.3 we provide a model that explicitly accommodates a hierarchy of quantifiers of the sort envisaged here (proposition 4.9). And in that context we provided a model which satisfies K2 (on any interpretation of its quantifiers), and even the stronger $\mathrm{K}^{+}$. Note that these schemata now include instances that involve the new typed quantifiers that we have introduced to the language in this environment. It thus follows that for any interpretation of the quantifiers in K1, that version of $\mathrm{K} 1$ will be an instance of our schema $\mathrm{K} 2$ (and a similar relation holds between $\mathrm{K}^{+}$and $\mathrm{K}^{+}$). As for $\mathrm{K} 3$ and $\mathrm{K}^{+}$there will be a different version of the principle for each quantifier, which is also validated in the model described in the proof of proposition 4.9.

Given these results, it is clear enough that as far as Prior-Kaplan puzzles are concerned there is no immediate benefit to ramifying both the quantifiers and the attitudes. Indeed, the dual ramification strategy would seem to have significant costs in this setting, viz K1-K3, after all if one merely ramifies quantifiers one can easily write down K1-K3 allowing that it is governed by the standard convention that 'thinks' has a uniform interpretation. But once one has ramified the attitudes, an endorsement of K1 appears much more problematic. Of course there may be other philosophical motivations for a more thorough going ramification. However considerations pertaining to the Prior-Kaplan paradoxes seem very weak in this regard.

\section{Concluding Thoughts}

Williamson is operating within a Fregean higher-order logic that combines universal instantiation with the thesis of higher-order necessitism (articulated using higher-order correlates of the Barcan formulae). Indeed, universal instantiation, and especially one of its consequences, comprehension, is a key argumentative tool in defending necessitism. In that setting two strands of his thinking come together very naturally. First that existence doesn't modally come and go, and second, that existence comes cheap. In this paper we have been concerned to explore frameworks in which there is no such natural alliance. On the one hand, we have looked at Fregean frameworks which reject universal instantiation. Such frameworks will inevitably reject the idea that existence comes 'cheap'. They may, however, accept necessitism, but not on the argumentative basis that Williamson offers.

One might wonder how these distinctions look in a Russellian framework. In a setting of thorough going ramification universal instantiation isn't even an option. We mentioned earlier that the Russellian might hope for a good facsimile of universal instantiation, one according to which a universally quantified claim entails all of its grammatical instances. Thus for example, if it's true that for all 1, , God wonders 2 whether $p$, no counterexample is provided by the sentence 'God doesn't wonder 2 whether he wonders 2 whether snow is white' because this not a grammatical sentence, since it violates the typing constraints. ${ }^{73}$ Nevertheless, given the cumulative hierarchy the proposed salvage of universal instantiation is unsuccessful. ${ }^{74}$ After all, an $n$th level expression can grammatically apply to anything that is a lower level, including levels more than one below. Suppose a third level predicate is true of all first level things, but

\footnotetext{
${ }^{73}$ Such a maneuvre surely would not constitute special pleading, for even the Fregean would not countenance 'is a horse is not a concept' as an instance of the first-order generalisation 'nothing is a concept'.

${ }^{74}$ This thought turns on the cumulative aspect of the hierarchy, but as noted in footnote 55, it's hard to properly distinguish the the Fregean from the non-cumulative ramifier, since we wouldn't want the distinction to turn on very superficial syntactic categories.
} 
not true of all second level things: $\forall x_{1} F_{3} x_{1} \wedge \exists y_{2} \neg F_{3} y_{2}$. Suppose that $t_{2}$ denotes a second level object that is not $F_{3}$, so that $\neg F_{3} t_{2}$. Then $F_{3} t_{2}$ is grammatical, but one cannot infer it from $\forall x_{1} F_{3} x_{1}$, thus universal instantiation even construed in the way just described fails. In short, universal instantiation is a bad idea for the Russellian, even if one's ramification is maximally thorough going. And since the idea that existence comes cheap is tightly allied to existential generalisation, a companion principle to universal instantiation, the Russellian necessitist cannot be motivated by that idea, nor by the logical principles that subserve it.

We've been exploring frameworks, Russellian and Fregean, that unlike Williamson disavow universal instantiation but which like Williamson may still endorse necessitist principles for each quantifier. To give things focus, we've been considering a particular cluster of motivations for rejecting universal instantiation, namely the desire to maintain the truth, or at least the logical coherence, of principles like K1-K3. Our exploration has however been rather pessimistic - although we hope instructive - and our own inclination is to take Prior's result at face value, and to reject principles like K1-K3 on that basis. There still remains the question of how to implement that discovery. Suppose on the heels of Prior's principle we grudgingly accept that it could never be that Tim Williamson seems to be saying that everything Tim Williamson seems to be seems to be saying is false, without there being something else Tim Williamson seems to be saying. It would nevertheless be unsatisfactory to suppose that if Tim Williamson at the point of death had never spoken, and was on the verge of seeming to be saying that everything he's seemed to say is false, then logic would somehow clamp his larynx, or else logic would somehow prolong his life until he seem to say something else, or render his audience deaf, and so on. In this connection, certain of the other puzzles discussed in this paper are suggestive, and may force us to grudgingly accept at least some aspects of the ramified vision. In particular, we may be forced to accept a family of thinking relations, that may take on at least some of the structural features of the Russellian hierarchy, and we may offer a diagnosis of our initial confusion in terms of our blindness to the proliferation of such relations. That said, it's a substantial further step to impose syntactic constraints typical of the standard ramifier. And even more importantly, it remains far from clear whether there is important philosophical work done by ramifying the quantifiers themselves - existence may yet come cheap.

\section{References}

[1] Andrew Bacon. Radical anti-disquotationalism. MS.

[2] Andrew Bacon. Vagueness at every order. MS.

[3] Thomas Bradwardine. Insolubilia. Peeters, 2010.

[4] Alonzo Church. Comparison of russell's resolution of the semantical antinomies with that of tarski. Journal of Symbolic Logic, 41(4):747-760, 1976.

[5] Martin Davies. Meaning, Quantification, Necessity: Themes in Philosophical Logic. Routledge \& Kegan Paul, 1981.

[6] John Divers. Possible Worlds. Routledge, 2006.

[7] Cian Dorr. Propositional profusion and the liar. MS.

[8] Michael Glanzberg. The liar in context. Philosophical Studies, 103(3):217-251, 2001. 
[9] J. H. Harris. What's so logical about the "logical" axioms? Studia Logica, 41(2-3):159-171, 1982.

[10] David Kaplan. Demonstratives. In Joseph Almog and John Perry and Howard Wettstein, editor, Themes From Kaplan, pages 481-563. Oxford University Press, 1977.

[11] David Kaplan. A problem in possible worlds semantics. In Walter Sinnott-Armstrong and Diana Raffman and Nicholas Asher, editor, Modality, Morality and Belief: Essays in Honor of Ruth Barcan Marcus, pages 41-52. Cambridge University Press, 1995.

[12] Saul A. Kripke. A puzzle about time and thought. In Saul A. Kripke, editor, Philosophical Troubles. Collected Papers Vol I. Oxford University Press, 2011.

[13] David K. Lewis. On the Plurality of Worlds. Blackwell Publishers, 1986.

[14] Kris McDaniel. Ways of being. In David John Chalmers, David Manley, and Ryan Wasserman, editors, Metametaphysics: New Essays on the Foundations of Ontology. Oxford University Press, 2009.

[15] Vann McGee. Everything. In Gila Sher and Richard L. Tieszen, editor, Between Logic and Intuition: Essays in Honor of Charles Parsons, pages 54-78. Cambridge University Press, 2000 .

[16] John Myhill. A refutation of an unjustified attack on the axiom of reducibility. In Bertrand Russell and George Washington Roberts, editor, Bertrand Russell Memorial Volume, pages 81-90. Humanities Press, 1979.

[17] Charles Parsons. The liar paradox. Journal of Philosophical Logic, 3(4):381-412, 1974.

[18] A. N. Prior. Modality and quantification in s5. Journal of Symbolic Logic, 21(1):60-62, 1956.

[19] A. N. Prior. On a family of paradoxes. Notre Dame Journal of Formal Logic, 2(1):16-32, 1961.

[20] A. N. Prior. Worlds, Times, and Selves. Duckworth, 1977.

[21] Frank Plumpton Ramsey. The Foundations of Mathematics and Other Logical Essays. Paterson, N.J.,Littlefield, Adams, 1960.

[22] Bertrand Russell. Mathematical logic as based on the theory of types. American Journal of Mathematics, 30(3):222-262, 1908.

[23] B. H. Slater. Prior's analytic. Analysis, 46(2):76-81, 1986.

[24] Nicholas J. J. Smith. Semantic regularity and the liar paradox. The Monist, 89(1):178-202, 2006.

[25] Alfred Tarski. The concept of truth in formalized languages. In A. Tarski, editor, Logic, Semantics, Metamathematics, pages 152-278. Oxford University Press, 1936.

[26] Dustin Tucker and Richmond H. Thomason. Paradoxes of intensionality. Review of Symbolic Logic, 4(3):394-411, 2011. 
[27] Gila Sher and Richard L. Tieszen, editor. Between Logic and Intuition: Essays in Honor of Charles Parsons. Cambridge University Press, 2000.

[28] Agustín Rayo and Gabriel Uzquiano. Toward a theory of second-order consequence. Notre Dame Journal of Formal Logic, 40(3):315-325, 1999.

[29] Gabriel Uzquiano. A neglected resolution of russell's paradox of propositions. Review of Symbolic Logic, 8(2):328-344, 2015.

[30] Agustín Rayo and Timothy Williamson. A completeness theorem for unrestricted firstorder languages. In Jc Beall, editor, Liars and Heaps. Oxford University Press, 2003.

[31] Timothy Williamson. Equivocation and existence. Proceedings of the Aristotelian Society, 88(n/a):109-127, 1988.

[32] Timothy Williamson. Bare possibilia. Erkenntnis, 48(2/3):257-73, 1998.

[33] Timothy Williamson. Everything. Philosophical Perspectives, 17(1):415-465, 2003.

[34] Timothy Williamson. Modal Logic as Metaphysics. Oxford University Press, 2013. 